\title{
Rayleigh scattering and disorder-induced mixing of polarizations in amorphous solids at the nanoscale: 1-octyl-3-methylimidazolium chloride glass
}

\author{
M. G. Izzo, ${ }^{1,2, *}$ B. Wehinger, ${ }^{3,4}$ S. Cazzato, ${ }^{5,6}$ A. Matic, ${ }^{6}$ C. Masciovecchio, ${ }^{7}$ A. Gessini, ${ }^{7}$ and G. Ruocco $\odot^{5,2}$ \\ ${ }^{1}$ Universitá degli studi di Roma "La Sapienza," Dipartimento di Ingegneria Informatica Automatica e Gestionale Antonio Ruberti, \\ Via Ariosto, 00185 Roma, Italy \\ ${ }^{2}$ Istituto Italiano di Tecnologia-Center for Life Nanoscience, Viale Regina Elena, 29100161 Roma, Italy \\ ${ }^{3}$ Department of Quantum Matter Physics, University of Geneva, 24 Quai Ernest Ansermet, 1211 Genève 4, Switzerland \\ ${ }^{4}$ Department of Molecular Sciences and Nanosystems, Ca' Foscari University of Venice, 30172 Venezia Mestre, Italy \\ ${ }^{5}$ Universitá degli studi di Roma "La Sapienza," Dipartimento di Fisica, Piazzale Aldo Moro 5, 00185 Roma, Italy \\ ${ }^{6}$ Department of Applied Physics, Chalmers University of Technology, Maskingränd 2, 41258 Gothenburg, Sweden \\ ${ }^{7}$ Sincrotrone Trieste S.C.p.A., S.S. 14 km 163,5 in AREA Science Park, I-34012 Basovizza, Italy
}

(Received 31 May 2017; revised 15 November 2020; accepted 17 November 2020; published 23 December 2020)

\begin{abstract}
Acousticlike excitations in topologically disordered media at mesocale/nanoscale present anomalous features with respect to the Debye's theory. The so-called Rayleigh scattering manifests in a strong increase of the attenuation of the acousticlike excitations and a softening of the phase velocity with respect to its continuum limit value. Mean field models developed in the random media theory framework can successfully predict the occurrence, at the proper length scale, of Rayleigh scattering. The overall attenuation in the Rayleigh region is, however, underestimated. In the framework of random media theory we developed an analytical model, which permits a quantitative description of the acousticlike excitations in topological glasses in the whole first pseudo-Brillouin zone. The underestimation of the Rayleigh scattering is avoided and, importantly, the model allows to account also for the polarization properties of the acousticlike excitations. In a three-dimensional medium an acoustic wave is characterized by its phase velocity, intensity, and polarization. Rayleigh scattering emphasizes how the topological disorder affects the first two properties. The topological disorder is, however, expected to influence also the third one. In common with the Rayleigh scattering, hallmarks possibly related to the mixing of polarizations have been traced in different classes of amorphous solids at nanoscale. The quantitative theoretical approach developed permits to demonstrate how the mixing of polarizations generates a distinctive feature in the dynamic structure factor of amorphous solids. The modeling capability of the proposed mean field theory is tested on glassy 1-octyl-3-methylimidazolium chloride, whose spatial distribution of the elastic moduli is well assessed and can be experimentally characterized. Contrast between theoretical and experimental features for the selected glass reveals an excellent agreement. The mean field approach we present retains a certain degree of generality and can be possibly extended to different stochastic media or different wave fields.
\end{abstract}

DOI: 10.1103/PhysRevB.102.214309

The modeling of the acoustic dynamics properties in amorphous solids at mesoscale/nanoscale is linked to the understanding of the origin of their macroscopic anomalies, such as the hump in the specific heat of glasses at $\sim 10 \mathrm{~K}[1]$ and the excess over the Debye level of the vibrational density of states (VDOS) at energies of few meV, called boson peak (BP) [2-7]. Several theoretical models have been proposed, based on different approaches. The so-called soft potential model $[4,8,9]$, an extension of the well-known tunneling two-level systems model, focuses on the presence, among the normal modes of an amorphous solid, of localized soft vibrations, correlated to the aforementioned anomalous behavior. Recently, the use of numerical methods based on molecular dynamics (MD) simulations allowed the observation and exact characterization of the localized soft modes by an atomistic

*Corresponding author: izzo@diag.uniroma1.it; mizzo@sissa.it approach [10-18]. A mean field approach is instead exploited in the models developed in the random media theory (RMT) framework, e.g., the so-called heterogeneous elasticity theory [5,19-23]. They are based on the modeling of the amorphous solid as a random medium with spatial fluctuating local elastic moduli. Among mean field theories we recall also the theory of jamming $[10,24]$, where the concept of marginal stability plays a central role.

In light or inelastic x-ray/neutron scattering experiments (IXS/INS) a plane wave with (transferred) momentum $(q)$ and energy $(E)$, or frequency $(\omega)$ being $E=\hbar \omega$, is initially excited in the sample. In crystals these excitations coincide with the normal modes, thus inelastic scattering experiments allow to selectively excite a single normal mode. This is not the case for amorphous solids, where, however, the acousticlike excitations generated by the interaction with the source can yet be expressed in the normal modes basis as a superposition of normal modes. IXS and INS techniques allow to experimentally 
access the so-called dynamic structure factor $S(q, E)$, whose expression as a superposition of the normal modes of the amorphous solid is provided, among others, in Refs. [25-27]. The normal modes can be derived by numerically solving the eigenvalue problem of the so-called dynamical matrix, i.e., the Hessian of the Hamiltonian calculated for the inherent structure obtained by MD simulations [10,12,27]. The class of localized soft modes between the normal modes of topological glasses carries particular relevance because localized soft modes are responsible for the $\omega^{4}$ trend (Rayleigh scattering) in the low- $\omega$ region of the VDOS [10-18], universally observed in glasses in contrast with the Debye law holding for crystals. Localized soft modes are non-Goldstone modes characterized by a small value of the so-called participation ratio [12], which establishes their local character, and a low value of their related eigenfrequency. The presence of localized soft modes in glasses was formerly emphasized by INS experiments on vitreous silica by exploiting a comparison with analytical results obtained for different polymorphic crystalline forms of $\mathrm{SiO}_{2}[28,29]$.

In place of the atomistic approach described above, mean field RMT models can be used in order to achieve an analytical representation of $S(q, E)$ of amorphous solids. At the expense of an exact assessment of normal modes, mean field RMTs allow an analytical evaluation of $S(q, E)$ based on a restricted number of key quantities. RMT relies on the observation that disorder on nanoscopic scale generates an inhomogeneous spatial distribution of the elastic moduli [3-5,19-23,30]. Modeling the amorphous solid as a random medium characterized by spatial elastic moduli fluctuations provides then the basis for the definition of a stochastic Helmholtz equation and of a related Dyson equation [3,5,19-22,31-34]. A statistical description of the spatial distribution of the local elastic moduli is obtained by defining the spatial correlation function of their fluctuations with respect to the average value on the system's volume [3,5,19-23,30]. In the case of randomly distributed spatial fluctuations, the correlation function is an exponential decay with decay constant $a$, equal to the average radius of spatial domains where the local elastic moduli remain roughly constant (so-called inhomogeneity or heterogeneity domains) [3,19-21,23,31,32,35]. A random distribution of local elastic moduli has been observed in a Lennard-Jones glass [11]. Long-range correlation has been also considered [30]. Interesting, MD simulations of a soft-spheres model [13] show how an amorphization transition is always accompanied by the simultaneous setting up of spatial elastic moduli fluctuations and appearance of localized soft modes among the normal modes. These results provide a framework for a link between the numerical atomistic approach and the mean field RMT. Similarly, in Ref. [36] it is established a connection between the so-called atomistic Euclidean random matrix model [37] and the self-consistent Born approximation (SCBA), a mean field model developed in the RMT framework, which will be discussed in the following.

Acousticlike excitations in amorphous solids at mesocale/nanoscale show an anomalous behavior with respect to the Debye predictions for crystals [2-5,22,23,30,3843]. The so-called Rayleigh scattering generates a softening of the phase velocity with respect to the macroscopic value and a strong increase of the attenua- tion, which shows the typical fourth-power $q$ dependence. This reflects on the aforementioned $\omega^{4}$ trend observed in the low- $\omega$ region of the VDOS. This behavior is almost universally observed in amorphous solids [5,10-18,22,30,38-43]. The modeling capability of Rayleigh scattering has been thus considered a valuable testing ground for theoretical models. Mean field RMTs present in literature can correctly reproduce the Rayleigh scattering specific trend with the proper related length scales. The overall strength of the attenuation induced by Rayleigh scattering is, however, in most cases underestimated [10,11,38,42-44]. By introducing an approximate solution of the Dyson equation, the so-called generalized Born approximation (GBA), we obtained a RMT model, which, in addition to the aforementioned results of previous RMTs, allows (i) to obtain a feasible strength of the attenuation in the Rayleigh scattering regime, avoiding underestimation; (ii) to account for the mixing of polarizations of acousticlike excitations in the first pseudo-Brillouin zone. These results represent an advance in both qualitative and quantitative modeling of acousticlike excitations of amorphous solids in the RMT framework. An acoustic wave traveling in a three-dimensional medium is characterized by its phase velocity, amplitude, and polarization. The presence of topological disorder is thus expected to affect all these properties. The Rayleigh scattering highlights how disorder affects the first two. The mixing of polarizations in a local elastically heterogeneous medium is qualitatively predicted by the elementary elasticity theory, which states that a purely polarized wave impinging on an interface between two different elastic media shall be transformed in waves with mixed polarization [45]. Features in $S(q, E)$ possibly attributable to acoustic excitations with mixed polarization have been observed by IXS or INS experiments [46-54], as well as by MD simulations [55-58] in several topologically disordered systems in the first pseudo-Brillouin zone, with a $q$ onset typically $\sim 5 \mathrm{~nm}^{-1}$. Despite this, one meets the lacking of analytical models addressing the occurrence of disorder-induced mixing of polarizations, which has, furthermore, never been related to the Rayleigh scattering in the RMT framework. We propose to cope with these shortcomings.

Inherent limitations of RMT models arise because, on the state of art, the Dyson equation can be solved only by recourse to approximation methods, which hold in a limited $q$ or $E$ range [5,19-22,31-34,59]. The Rayleigh scattering and the mixing of polarizations occur in different $q$ regions. The concomitance of these facts can possibly avoid the attainment of a unified theory. The Rayleigh scattering appears when $q$ is much lower than the inverse of $a$ whereas the coupling between longitudinal and transverse excitations is maximum when $q$ becomes comparable to $a^{-1}$ [31]. It can be shown the validity of the GBA up to $q=O\left(a^{-1}\right)$. In several glasses $a$ is of the order of $\mathrm{nm}[11,60-63]$, setting the region of validity of the GBA approximately coincident with the first pseudoBrillouin zone [43,47-49,51,52].

It is worth to stress that while the GBA proved to seize significant properties of acousticlike excitations in elastically heterogeneous media, as outlined in this paper, its cardinal mathematical structure and the nature of the underlying physical mechanisms (scattering) addressing the phenomena it aims to model could allow a useful exploiting of the GBA in 
different fields. On the other hand, mean field approaches in RMT framework in order to describe wave fields in stochastic media, such as the coherent potential approximation [64,65], are not limited to the case of elastically heterogeneous media, encompassing, among others, randomly dilute magnets [66], random Kondo lattice [67], disordered strongly correlated systems [68], and disordered semiconductors with spatially correlated disorder [69]. The SCBA itself has been formerly introduced independently in different fields of physics: scattering of electrons in disordered media [70,71], elastic wave propagation in topologically disordered systems [72], and electron-phonon interaction [73,74].

The GBA is tested on longitudinal acoustic dynamics of glassy 1-octyl-3-methylimidazolium chloride, [C8MIM]Cl. This latter belongs to the class of the so-called roomtemperature ionic liquids (ILs). The wave-vector-resolved acoustic dynamics features of glassy [C8MIM]Cl have been probed by IXS. In particular, the acoustic dynamics of a glass in the wave-vector range where the mixing of polarization onsets has been characterized in this work with unprecedented precision. Proper INS measurements yield the corresponding VDOS. The choice of glassy [C8MIM]Cl in order to test the GBA has been directed by the two following grounds. (i) In glassy ILs the elastic heterogeneity is defined by the alternation of polar (stiffer) and nonpolar (softer) domains [60,75-81], which remains well defined in ILs with sufficiently long alkyl chains. The (elastic) heterogeneity domains thus turn out to be clearly bordered. (ii) In ILs there exists a correlation between elastic heterogeneity and local topology [60]. The occurrences in points (i) and (ii) make possible the experimental characterization of the spatial distribution of the local elastic moduli and, in particular, of the value of $a$, which can be thus fix by unrelated measurements instead to be an adjustable parameter of the theory. The GBA is particularly sensitive to the value of $a$ because it determines the length scale where the Rayleigh scattering is toned down and the mixing of polarizations onsets. To fix the value of $a$ thus can avoid possible tautology when testing the GBA. The local elastic moduli can, otherwise, be characterized by MD simulations [13,61-63]. In Ref. [82] we show the mathematical coherence of the GBA and discuss its domain of validity in the kinematic plane. Approximation methods developed in the RMT framework usually rely upon the so-called perturbative series, formally representing the so-called self-energy (see the discussion in Sec. II). Because in most real cases even establishing the criteria of convergence of the perturbative series is not possible [33,34], the evaluation of the error associated to a given approximation can be unwieldy. Usually, necessary rather than sufficient conditions of validity can be set out, as it is the case of the Born approximation [33]. The comparison with experimental characterization of real systems becomes thus a valuable indication of the appropriateness of the theory. The comparison between experimental and GBA theoretical features provided in this work is excellent.

The paper is organized as follows. Section I contains a description of the experiments, of a phenomenological data analysis based on damped harmonic oscillator (DHO) functions, discusses the experimental results and compares them with the characterization of several other amorphous solids available in literature. Section II introduces the GBA, discusses its framing in the RMT, and explains how theoretical results are produced. Section III shows the general understanding of longitudinal and transverse acoustic dynamics of amorphous solids on nanoscopic region provided by GBA, while discussing how it can quantitatively reproduce experimental results for glassy $[\mathrm{C} 8 \mathrm{MIM}] \mathrm{Cl}$. Concluding remarks are presented in Sec. IV. Appendix A contains specifics of GBA calculations. Appendix B discusses the coupling of acousticlike excitations, modeled by GBA, with pure molecular modes.

\section{EXPERIMENTAL CHARACTERIZATION OF THE VIBRATIONAL DYNAMICS IN A LOCAL ELASTICALLY HETEROGENEOUS MEDIUM}

The local topology at nanoscale of most ILs has been largely attested and characterized by small-angle $\mathrm{x}$-ray or neutron scattering measurements and by MD simulations [75-81]. The static structure factor $S(q)$ of ILs with sufficiently long alkyl chains is characterized by a first sharp diffraction peak (FSDP) at a characteristic wave vector $q_{\mathrm{FSDP}}$. Upon definition of the ring centers and anions as polar entities and the alkyl chains as nonpolar ones, the segregation of the alkyl chains results in a local structure formed by an alternation of polar and nonpolar domains [79]. As proved by neutron and $\mathrm{x}$-ray scattering experiments $[75,77,80]$, the FSDP is related to the scattering signal coming from those anions that are on the side of the charged imidazolium ring belonging to cations that have opposite alkyl tails (see the pictorial representation in Fig. 5). The characteristic length scale $2 \tilde{a}=$ $2 \pi / q_{\text {FSDP }}$ gives thus an estimation of the diameter of nonpolar domains [75]. Since intermolecular forces acting in polar and nonpolar regions are of different nature, it is possible to assume that to the heterogeneous local structure it corresponds a heterogeneous spatial distribution of the local elastic constants. This inference is confirmed by the MD simulations results presented in Ref. [60], which provide a quantitative measure of local elastic moduli in ILs [C8MIM][NO3] and [CnMIM][PF6], $n=2,4,6,8$. The correlation between the spatial distribution of the elastic moduli and polar and nonpolar segregation is therein proved. It is thus possible to assume that the size of nonpolar domains coincides with the one of elastic heterogeneity domains, i.e., $a=\tilde{a}$. Among the large class of ILs, [C8MIM]Cl has been selected because (1) $q_{\mathrm{FSDP}}$ matches the $q$ region typically probed by IXS thus making sure that both the $q$ regions where the Rayleigh scattering and the mixing of polarization respectively occurs will be probed [81]; (2) the width of the FSDP is sufficiently small to permit to safely neglect the distribution of the size of the heterogeneity domains around their average value, i.e., the value of $a$ is well defined. The presence of the FSDP can give rise, however, to features in $S(q, E)$, related to the so-called diffuse umklapp scattering [25,83-87], which, though having a different origin, are similar to those related to the mixing of polarizations. We, however, verify that the umklapp scattering contribution is negligible in the $q$ region where the GBA apply, i.e., up to the edge of the first pseudo-Brillouin zone, and in particular at those $q$ 's where the mixing of polarizations onsets (see Supplemental Material [88]). These conditions make the experimental observations on the selected glass a reliable base for a genuine testing of the theory. 
The distinctive features of the vibrational dynamics of glassy $[\mathrm{C} 8 \mathrm{MIM}] \mathrm{Cl}$ are experimentally determined through IXS and INS. A preliminary data analysis of IXS results, based on a phenomenological model including one or two DHO functions, largely exploited in literature, is introduced. This protocol allows to identify distinctive features of the experimentally determined acoustic dynamics of glassy $[\mathrm{C} 8 \mathrm{MIM}] \mathrm{Cl}$, irrespective of any underlying theoretical model. Hallmarks of the Rayleigh scattering and of the mixing of polarization are highlighted. The phenomenological data analysis further allows the comparison between the characteristics of the acoustic dynamics of glassy [C8MIM] $\mathrm{Cl}$ and of other classes of glass, available in literature, brought out by exploiting the same phenomenological model. The comparison underlines a general qualitative agreement.

\section{A. Inelastic x-ray scattering experiment}

The IXS experiment was carried out at the ID28 beamline of the European Synchrotron Radiation Facility (ESRF). The data were collected in the $q$ range $[1-15] \mathrm{nm}^{-1}$ with a $q$ step of about $0.4 \mathrm{~nm}^{-1}$. The experiment was performed at $23.725 \mathrm{keV}$, using the silicon $(12,12,12)$ reflection, which provides an overall energy resolution of about $1.4 \mathrm{meV}$ (FWHM), determined from a plexiglass slab at $T=10 \mathrm{~K}$. The $q$ resolution was set to $0.18 \mathrm{~nm}^{-1}$ for the first $q$ points (i.e., $1.0 \mathrm{~nm}^{-1}, 1.4 \mathrm{~nm}^{-1}, 1.8 \mathrm{~nm}^{-1}$ ) and fixed to $0.37 \mathrm{~nm}^{-1}$ for the remaining $q$ 's. The energy spectra were collected in the -30 to $30 \mathrm{meV}$ range. The sample cell was an $\mathrm{Al}$ tube of 5 -mm length capped with two oriented monocrystal diamond windows ( $0.5 \mathrm{~mm}$ thick). The [C8MIM]Cl sample was loaded in the sample cell in an Ar-filled glove box to avoid water contamination. A cryostat was used to cool the sample down to the glassy state. The background contribution from the sample environment was measured in the same experimental configuration as the sample and subtracted from the data, thus obtaining the sample signal $I(q, E)$. This latter provides a measure of the dynamic structure factor related to longitudinal acoustic dynamics $S_{L}(q, E)$, via the expression

$$
I(q, E)=A(q)\left[E \frac{n(E)+1}{k_{B} T} S_{L}(q, E)\right] \otimes R(E)+c(q),
$$

where $E=\hbar \omega$ is the exchanged energy between the probe and the sample, $\omega$ is the exchanged frequency, $\hbar$ is the reduced Planck constant, $n(E)$ is the Bose factor, $R(E)$ is the experimental energy resolution and $\otimes$ represents the convolution operation. The adjustable parameters $A(q)$ and $c(q)$ are, respectively, an overall intensity factor and a background.

\section{B. Inelastic neutron scattering experiment}

The INS experiment was performed with the MARI spectrometer at the ISIS spallation neutron source in Rutherford Appleton Laboratory. The sample was loaded in an $\mathrm{Al}$ made cylindrical annular can with a thickness corresponding to a sample transmission $T=0.9$. The incident neutron energy was $15 \mathrm{meV}$. The temperature of the sample was tuned by a cryostat. The empty cell scattering $I_{\mathrm{EC}}(q, E)$ was measured under the same sample experimental conditions. The sample signal $I(q, E)$ is obtained from the total signal $I_{t}(q, E)$, through the relationship $I(q, E)=I_{t}(q, E)-$ $T I_{\mathrm{EC}}(q, E)$. Multiple scattering and self-absorption are as- (a)

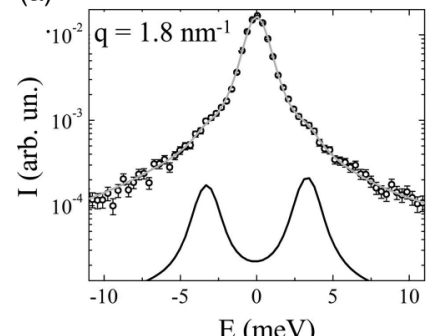

(c)

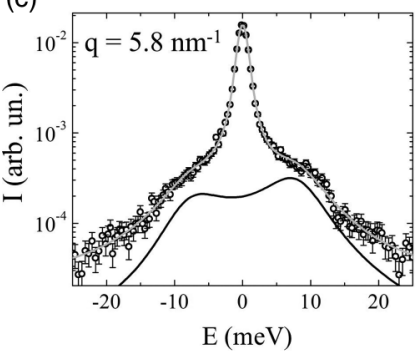

(b)

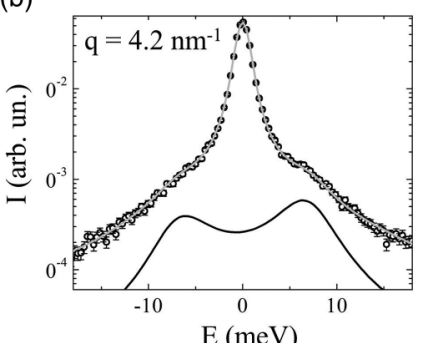

(d)

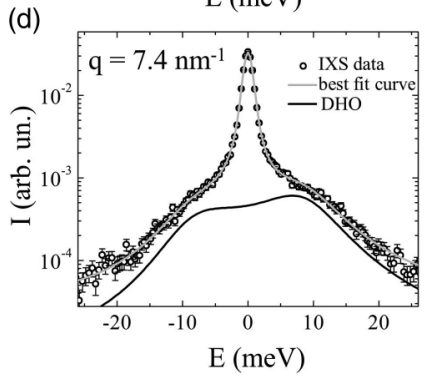

FIG. 1. (a)-(d) IXS spectra of glassy [C8MIM]Cl at $T=176 \mathrm{~K}$ for selected $q$ values (black circles with error bars), best-fit curves obtained by 1-DHO fitting model (gray lines) and inelastic components of the best-fit curves, i.e., DHO function convoluted with the experimental resolution (black lines).

sumed to be negligible for the given sample geometry and transmission coefficient. For [C8MIM]Cl the incoherent neutron scattering cross section $\sigma_{\text {incoh }}$ is much larger than the coherent one $\sigma_{\text {coh }}$, being $\frac{\sigma_{\text {coh }}}{\sigma_{\text {inch }}}=0.07$. The measured data are integrated over $q$ in the largest range available from the experimental configuration, i.e., $20<q<40 \mathrm{~nm}^{-1}$. It is thus obtained the averaged VDOS, neutron weighted over the atomic species. The reduced VDOS, $\frac{g(E)}{E^{2}}$, is obtained from the integrated signal $I(E)$ through the relationship $\frac{g(E)}{E^{2}}=$ $A \frac{I(E)}{E[n(E)+1]}$, where $A$ is an intensity factor.

Experimental characterization of longitudinal acoustic dynamics and VDOS of [C8MIM]Cl at $T=176 \mathrm{~K}\left(T_{g}=214 \mathrm{~K}\right)$ was achieved, respectively, by IXS and INS. Figure 1 shows IXS spectra for selected $q$ values. High-quality data were obtained at all wave vectors. An inelastic contribution is detectable in all the spectra, except for those corresponding to $q$ close to $q_{\mathrm{FSDP}}$, where the spectrum is dominated by the strong elastic signal. In the phenomenological data analysis discussed in this section, $S_{L}(q, E)$ is modeled by a linear combination of DHO functions, i.e.,

$$
S_{L}(q, E)=\sum_{n} \frac{\tilde{I}_{L(n)}(q) \Gamma_{L(n)}(q) \Omega_{L(n)}^{2}(q)}{\left[\Omega_{L(n)}^{2}(q)-E^{2}\right]^{2}+E^{2} \Gamma_{L(n)}^{2}(q)},
$$

where $n=1$ for the 1-DHO fitting model and $n=1,2$ for the 2-DHO fitting model. $\tilde{I}_{L(n)}=I_{L(n)} / I_{L(1)}$ is the relative intensity of the $n$th DHO function with respect to the first. A zerocentered delta function $I_{0}(q) \delta(E)$ is added to $S_{L}(q, E)$ in order to account for the elastic scattering signal observed in the experimental spectra. $I_{0}(q), \tilde{I}_{n}(q), \Omega_{L(n)}(q), \Gamma_{L(n)}(q)$ are adjustable parameters. At first, a fit of all the spectra acquired is performed by using a single DHO function as model function (1-DHO fitting model). Such a protocol provides a measure of the characteristic energy and broadening (attenuation) of the 


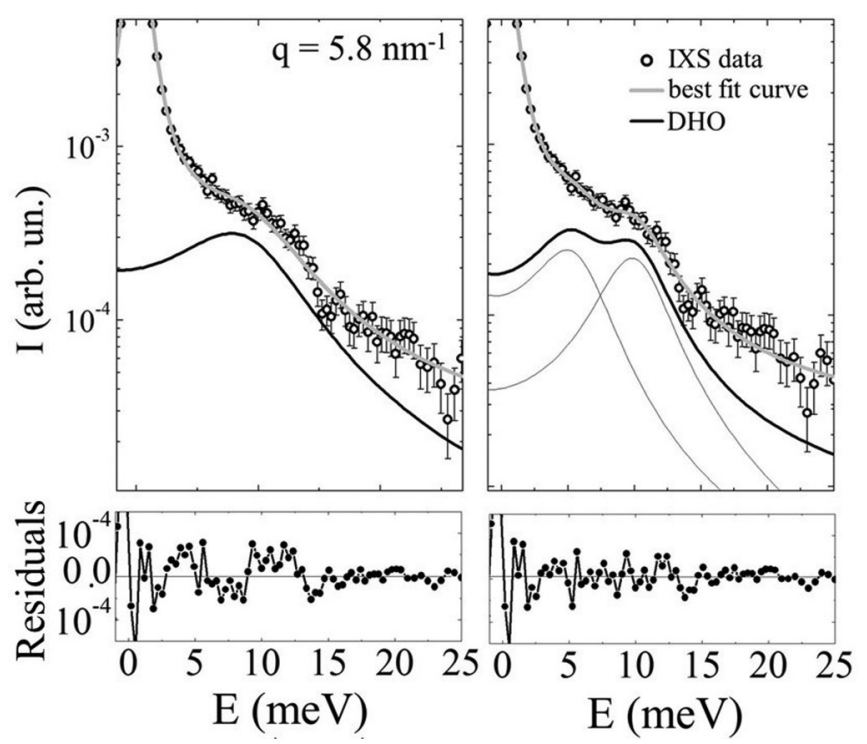

FIG. 2. Detail of a representative IXS spectrum (black circles with error bars) in the high- $q$ region. Best-fit curves (gray lines) and inelastic components (black lines) obtained for 1-DHO fitting model (right panel) and 2-DHO fitting model (left panel) are shown. Corresponding fit residuals are shown in the bottom panels.

longitudinal acoustic excitation for each $q$, respectively $\Omega_{L}(q)$ and $\Gamma_{L}(q)$. The values of $\Omega_{L}(q)$ and $\Gamma_{L}(q)$ with the corresponding error bars obtained by exploiting the 1-DHO fitting model are displayed in Figs. 3(a) and 3(c) by open circles. The high quality of the IXS data acquired permits to assign a value to $\Omega_{L}$ and $\Gamma_{L}$ even at small values of $q$, in the interval $1-2 \mathrm{~nm}^{-1}$. In the high- $q$ region $\left(q>5 \mathrm{~nm}^{-1}\right)$ an additional feature in the inelastic wings of the IXS spectrum is observed, in agreement with experimental observation in several other classes of glass [46-58]. Similar features are commonly related to "projection" of transverse into longitudinal dynamics. To empirically account for the presence of this extra feature, the inelastic part of the spectrum can be fitted with a model function that includes two DHO functions (2-DHO fitting model). Figure 2 shows a magnification of the inelastic component of an IXS spectrum acquired at $q>5 \mathrm{~nm}^{-1}$. Best-fit curves and fit residuals corresponding to 1-DHO and 2-DHO fitting models are included for comparison. Inspection of fit residuals corresponding to $1-\mathrm{DHO}$ fitting model shows the presence of structured features revealing indeed inadequacy in the choice of this fitting model function. Figures 3(a) and 3(c) show the best-fit values of $\Omega_{L}$ and $\Gamma_{L}$ corresponding to each one of the two DHO functions composing the fitting model function for $q>5 \mathrm{~nm}^{-1}$, displayed respectively by blue and green full circles. We will consider both best-fit results obtained by fitting with the 1-DHO fitting model in the whole measured $q$ region and with the 2-DHO fitting model in the only high- $q$ region. The trend of $\Omega_{L}(q)$ and $\Gamma_{L}(q)$ obtained by the 1-DHO fitting model presents at $q \sim a^{-1}$ features that can be related to the mixing of polarizations, described in points (ii) and (iii) in the following and examined in Sec. III. As emphasized in Sec. III the IXS spectra in the whole experimentally accessed $q$ range can be fully reproduced by GBA-based calculations. This makes the fitting with 1-DHO
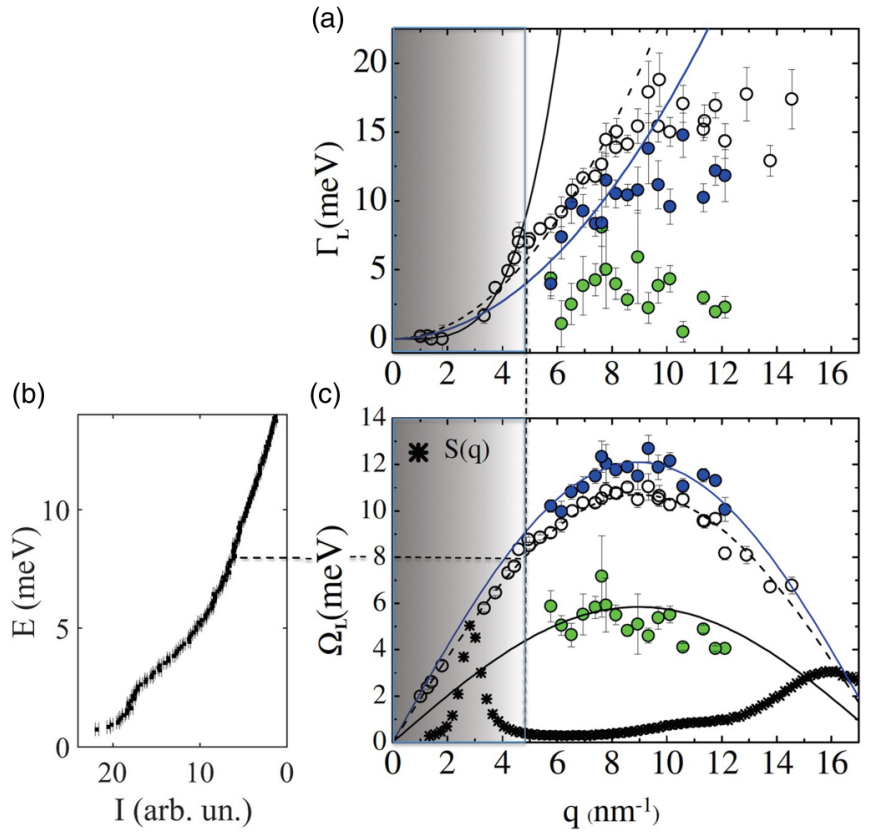

FIG. 3. (a) Broadening $\left(\Gamma_{L}\right)$ as a function of $q$ derived from the fitting of the IXS data with 1-DHO fitting model (open circle) and 2-DHO fitting model (blue and green circles). Full black line shows the $q^{4}$ trend reproducing the $\Gamma_{L}$ dispersion in the low- $q$ region. Blue and dashed black lines are guides to eye displaying the $q^{2}$ trend of the broadenings in the high- $q$ region. (b) Reduced VDOS, $g(E) / E^{2}$, obtained by INS measurements. (c) Characteristic energy $\left(\Omega_{L}\right)$ of the inelastic excitations as a function of $q$, obtained with 1-DHO fitting model (open circle) and 2-DHO fitting model in the high- $q$ region (blue and green circles). The dashed, blue, and black lines reproduce the dispersion curves of $\Omega_{L}$ 's. They are obtained by the sinusoidal function $c \frac{q_{0}}{\pi} \sin \left(\frac{q \pi}{q_{0}}\right)$ [98], where $c$ and $q_{0}$ are adjustable parameters. The x-ray-weighted static structure factor is shown (stars).

or 2-DHO model functions an experimentally convenient, yet theoretically unnecessary, phenomenological expedient.

Figure 3 shows $g(E) / E^{2}$ [Fig. 3(b)] and $S(q)$ [Fig. 3(c)], measured by $\mathrm{x}$-ray scattering. The reduced VDOS presents (1) a peak around $2 \mathrm{meV}$, referred to as the boson peak (BP) [89,90]; (2) a broad peaklike feature at higher energies, in the region between 7 and $12 \mathrm{meV}$, related to pure molecular modes, identified in Ref. [89] with the librational modes of the imidazolium ring, referred hereafter as intermolecular vibrational modes (IVMs). The static structure factor shows a FSDP at $q_{\mathrm{FSDP}}=2.8 \mathrm{~nm}^{-1}$, which is related to nanoscale segregation of the cations' alkyl chains [75,78]. This corresponds to a value of $a$ equal to $1.1 \mathrm{~nm}$. It has been shown that the short-range structure of glasses preserves a residual order that characterizes the long-range structure of crystals $[6,54]$ with the consequent occurrence of a pseudo-Brillouin zone and of a related bending of the dispersion of the characteristic energies of acousticlike excitations. Observation of Fig. 3(c) clearly points out the presence of the bending in the dispersion of the characteristic energies, allowing the identification of the pseudo-Brillouin zone. From the experimental results the edge of the first pseudo-Brillouin zone, where the maximum of the dispersion is observed, can be fixed at $\sim 9 \mathrm{~nm}^{-1}$, about half the value of the characteristic $q$ of the main peak in $S(q)$. 
Inspection of Fig. 3 permits to identify the following characteristics of the acoustic dynamics of glassy [C8MIM]Cl in the first pseudo-Brillouin zone: (i) in the low- $q$ region [gray-shaded region in Figs. 3(a) and 3(c)] features compatible with the Rayleigh scattering are observed. The increase of the acoustic attenuation following the $q^{4}$ trend is clearly observable. The softening of the phase velocity is better highlighted in Fig. 7, but it can be perceived, even if with lower clarity, also in the $q$ trend of $\Omega_{L}$ 's displayed in Fig. 3 . (ii) It exists a crossover in the wave-vector dispersion of $\Gamma_{L}$ at $q_{c}=4.8 \mathrm{~nm}^{-1}$ from a $q^{4}$ to a $q^{2}$ trend. We observe that $q_{c} \sim 2 q_{\mathrm{FSDP}} \sim 2 \pi / a$, i.e., the crossover appears at wave vectors related to the typical size of elastic heterogeneity domains. (iii) There is a kink at $q_{c}$ in the $\Omega_{L}$ dispersion. (iv) For $q>q_{c}$ the inelastic wings of $S_{L}(q, E)$ are composed by two features. Similar characteristics of the ones described in points (i)-(iv) are observed in literature in several other amorphous solids by IXS/INS experiments and MD simulations [5,11,22,38-43,46-49,51,55]. Among them, we point vitreous silica, which is usually promoted to a prototype of topological glasses. In order to clarify the origin of the low- $E$ feature observed in $S_{L}(q, E)$ for $q>q_{c}$ [see point (iv) above], we calculated the so-called diffuse umklapp scattering contribution to $S_{L}(q, E)$ by applying the protocols described in Refs. $[25,86]$ to glassy [C8MIM]Cl. Specifics are reported in the Supplemental Material [88]. The intensity of the diffuse umklapp scattering has local maxima around the peaks of $S(q)$. The presence of the FSDP requires thus the need to check if the umklapp scattering can contribute to the $S_{L}(q, E)$ for $q>q_{c}$, thus generating the observed extra feature. The results described in the Supplemental Material [88] emphasize, however, that the umklapp scattering contribution is negligible in the first pseudo-Brillouin zone and, in particular, such it is at $q \sim q_{c}$, where the regime specified in point (iv) onsets. This allows to exclude that in the first pseudo-Brillouin zone the extra feature is related to the diffuse umklapp scattering. Features in $S_{L}(q, E)$ similar to the ones described in point (iv) have been observed, at a $q$ onset close to $q_{c}$, in several classes of glass, among which, vitreous silica $[46,48]$, glassy $\mathrm{GeO}_{2}$ [51], glassy glycerol [47], glassy $\mathrm{SiSe}_{2}$ [49]. The $S(q)$ of these glasses shows any FSDP in $S(q)$ and the umklapp scattering is furthermore negligible in the $q$ region where the extra feature in $S_{L}(q, E)$ is observed [86]. Such an extra feature in $S_{L}(q, E)$ is in all these cases qualitatively attributed to the mixing of polarizations. For $q$ 's outside the first pseudo-Brillouin zone, i.e., for the largest $q$ 's accessed in the present IXS experiment, it must be, however, considered that the diffuse umklapp scattering contribution is not negligible (see Supplemental Material [88]). The GBA does not include the contribution of the diffuse umklapp scattering and, on the other hand, its validity is not attested for $q$ much larger than $a^{-1}$. This shall be taken into account when comparing GBA and experimental outcomes at $q \gtrsim 11 \mathrm{~nm}^{-1}$. For these $q$ values the use of the GBA to model the experimental data can actually be not well established. For $q$ values well outside the first pseudoBrillouin zone, the use of the incoherent approximation allows a reliable theoretical description of the experimentally determined $S_{L}(q, E)$ [48,86,91]. In the case of vitreous silica [48], e.g., it has been shown that the secondary peaklike feature observed in $S_{L}(q, E)$, in the first pseudo-Brillouin zone related to the mixing of polarizations, in the high- $q$ region, where the incoherent approximation can be applied, merges into an opticlike branch with frequency close to the one of the BP and related to the vibrational modes of $\mathrm{SiO}_{4}$ tetrahedra [28,29]. The modeling of $S_{L}(q, E)$ in this high- $q$ region [92] is beyond the scope of this paper.

We show in Sec. III how the behavior described in points (i)-(iv) can be quantitatively described by the proposed stochastic approach derived in the framework of the RMT, which accounts for the elastic heterogeneity of the disordered system. In particular, the features observed at $q$ of the order of or larger than $q_{c}$ in the GBA framework can be related to the mixing of polarizations.

It shall be noticed that the value of $\Omega_{L}\left(q_{c}\right)(\sim 8 \mathrm{meV})$ belongs to the energy range of the VDOS where it is observed the broad feature related to IVMs. Their characteristic energy is much larger than the one of the BP and they are not to be rated as localized soft modes introduced above. They are rather similar to pure molecular modes observed in molecular crystals. They can, however, possibly couple to the acousticlike excitations. We set in the following the polarization-dependent coupling parameter to zero. For sake of completeness, we consider in Appendix B a model where the coupling factor is different from zero. We, however, stress that by setting the coupling parameter to zero, the GBA allows, nevertheless, to achieve an exhaustive analytical modeling of the longitudinal acoustic dynamics of glassy $[\mathrm{C} 8 \mathrm{MIM}] \mathrm{Cl}$. Furthermore, since the effect of the coupling with IVM mostly affects the acousticlike excitations with characteristic energy matching the one of the IVM, exploiting approximation different from the GBA, such as the Born approximation or the SCBA, with a coupling parameter different from zero does not permit to obtain a comprehensive modeling of the longitudinal acoustic dynamics in the whole first pseudo-Brillouin zone (see Supplemental Material [88]).

\section{GENERALIZED BORN APPROXIMATION IN THE RANDOM MEDIA THEORY}

\section{A. Random media theory and generalized Born approximation}

Purpose of the RMT is to describe the space (r) and time $(t)$ evolution of the ensemble-averaged response of a spatial heterogeneous medium after the application of an external input in the space point $\mathbf{r}^{\prime}$. The system's response is formally expressed by the second-rank averaged Green's dyadic $\left\langle\mathbf{G}\left(\mathbf{r}, \mathbf{r}^{\prime}, t\right)\right\rangle$, solution of the corresponding Dyson equation. The brackets $\langle\ldots\rangle$ denote ensemble average. The bold font style displays a tensor. By virtue of the fluctuationdissipation theorem, the dynamic structure factors related to both longitudinal and transverse dynamics can be expressed as a function of the corresponding averaged Green's functions $\left\langle G_{L(T)}(\mathbf{q}, \omega)\right\rangle$, i.e., $S_{L(T)}(\mathbf{q}, \omega)=\frac{1}{\pi} \frac{q^{2}}{\omega} \operatorname{Im}\left\{\left\langle G_{L}(\mathbf{q}, \omega)\right\rangle\right\}$. The wave vectors $\mathbf{q}$ and $\omega$ are the conjugate Fourier variables of $\mathbf{r}$ and $t$, respectively. The spatial distribution of fluctuating quantities is statistically described by their space-correlation function. We let the only elastic tensor $\mathbf{C}(\mathbf{r})$ to fluctuate in space. A formal statement of the Dyson equation provides for the introduction of a self-energy or mass operator $\boldsymbol{\Sigma}(\mathbf{q}, \omega)$, which embeds all the information related to the system's 
inhomogeneity,

$$
\langle\mathbf{G}(\mathbf{q}, \omega)\rangle=\left[\mathbf{G}^{0}(\mathbf{q}, \omega)^{-1}-\mathbf{\Sigma}(\mathbf{q}, \omega)\right]^{-1},
$$

where $\mathbf{G}^{0}(\mathbf{q}, \omega)$ is the bare Green's dyadic describing the "bare" system in the absence of spatial fluctuations (see Appendix A). The self-energy can be formally cast by a perturbative Neuman-Liouville series expansion [33,34]. Through the introduction of the Feynman diagram technique it is possible to establish a correspondence between the terms composing the perturbative series expansion and the scattering events of the elastic perturbation in the medium [33]. Under the hypothesis of statistical homogeneity, truncation of the perturbative series expansion to the lowest nonzero order (first order) leads to the so-called Bourret or Born approximation [20,31-34,59]

$$
\begin{aligned}
\Sigma_{k \alpha}^{1}(\mathbf{q}, \omega)= & \hat{L}_{1 k \alpha i j} G_{i j}^{0}(\mathbf{q}, \omega) \int d^{3} s q_{\beta} q_{l} s_{\delta} s_{\gamma} \tilde{R}_{\gamma \alpha j l \beta k i \delta} \\
& \times(\mathbf{q}-\mathbf{s}) G_{i j}^{0}(\mathbf{s}, \omega) .
\end{aligned}
$$

Summation over repeated indices is assumed. The integral is extended to $\mathbb{R}^{3}$. The function $\tilde{R}_{\gamma \alpha j l \beta k i \delta}(\mathbf{q})$ is the Fourier transform of the correlation of the elastic tensor fluctuations $R_{\gamma \alpha j l \beta k i \delta}\left(\mathbf{r}=\mathbf{r}_{1}-\mathbf{r}_{2}\right)=\left\langle\delta C_{\gamma \alpha j l}\left(\mathbf{r}_{1}\right) \delta C_{\beta k i \delta}\left(\mathbf{r}_{2}\right)\right\rangle$, where $\delta \mathbf{C}$ states for a fluctuation of the elastic tensor with respect to its average value $\overline{\mathbf{C}}$. The self-energy in the Fourier space can thus be written as a convolution between the bare Green's dyadic and the Fourier transform of the space correlation of the elastic tensor fluctuations. Despite simplicity, the Born approximation imposes rather strong restrictions both on the intensity of the elastic moduli fluctuations per density $\epsilon^{2}$ and on the value of $\omega$ and $q$, which need to be small with respect to $a^{-1}$ [33]. Most of the phenomenology observed in real systems, including the Rayleigh scattering, can be qualitatively grasped even by the Born approximation [31]. In a vectorial framework it can even account for some features of acousticlike excitations related to the coupling between longitudinal and transverse dynamics [31]. However, to rely on this approximation did not permit us neither to quantitatively characterize the overall strength of the Rayleigh scattering nor to unravel the presence of a well-defined shoulder in $S_{L}(\mathbf{q}, \omega)$ related to the coupling of longitudinal with transverse acoustic dynamics [82], as instead attested by experimental and MD simulations' observation. The limited range of validity in the $q$ space of the Born approximation, which furthermore shifts to lower values of $q$ for larger value of $\epsilon^{2}$, what it is expected in the case of glasses, can affect its outcomes in particular when $q \sim a^{-1}$. In light of these observations, we choose to take into account the next order of approximation in the perturbative series expansion [33], thus obtaining the following expression for the self-energy:

$$
\Sigma_{k \alpha}(\mathbf{q}, \omega)=\hat{L}_{1 k \alpha i j}\left\langle G_{i j}(\mathbf{q}, \omega)\right\rangle^{1},
$$

where $\langle\mathbf{G}(\mathbf{q}, \omega)\rangle^{1}$ is the ensemble-averaged Green dyadic calculated in the Born approximation. On a physics ground the inclusion of second-order terms permits to describe multiple scattering events not covered by the Born approximation [82]. Second-order terms of the perturbative series are in the present approach accounted in an approximate form. Details are reported in the next paragraph. We thus introduce a method to derive an approximate expression for $\boldsymbol{\Sigma}(\mathbf{q}, \omega)$, which states corrective terms to the Born approximation in the framework of the perturbative series expansion. We refer to it as to a generalized Born approximation [82]. The mathematical coherence of the GBA and its validity at wave vectors of the order of $a^{-1}$ is discussed in Ref. [82]. Generalizations of the Born approximation have attracted interest in different fields [93-96]. In particular, depolarization effects in the scattering of electromagnetic waves by an isotropic random medium has been predicted by exploiting a second-order representation (with respect to terms of a Neumann iteration series) for the scattered intensity [95]. The results we obtained through the GBA, enforced by the above cited study, suggest that in order to properly describe the polarization properties, including the mixing of polarizations, of wave fields in isotropic random media, without necessarily restricting to the case of elastically heterogeneous media, second-order terms of the perturbative series expansion should be taken into account.

Before giving the details of the expression of the selfenergy stated by the GBA, we observe that a largely exploited model to characterize the Rayleigh scattering in glasses is the so-called self-consistent Born approximation (SCBA) $[3,5,19,21,22]$. Instead of looking to a suitable expression for the mass operator by truncating the Neumann-Liouville series, the stochastic equation describing the disordered system is replaced by an effective nonlinear deterministic equation. The SCBA or Kraichnan model [72] reads as

$$
\Sigma_{k \alpha}(\mathbf{q}, \omega)=\hat{L}_{1 k \alpha i j}\left\langle G_{i j}(\mathbf{q}, \omega)\right\rangle .
$$

Equation (6) together with Eq. (3) corresponds to successive self-consistent approximations of $\boldsymbol{\Sigma}(\mathbf{q}, \omega)$ and $\langle\mathbf{G}(\mathbf{q}, \omega)\rangle$. At the zeroth step it is $\langle\mathbf{G}(\mathbf{q}, \omega)\rangle=\mathbf{G}^{0}(\mathbf{q}, \omega)$. The first step of the iteration procedure thus corresponds to the Born approximation, whereas Eq. (5) corresponds to the second step. The SCBA is usually used in the Rayleigh region by neglecting the $q$ dependence of the self-energy, thus achieving an analytical expression $[5,11,19,22,42]$. Information about the polarization properties of the acousticlike excitations in topologically disordered systems carried from the SCBA can be hidden by the unfeasibility to achieve analytical results at $q \sim a^{-1}$, where the $q$ dependence of the self-energy cannot be ignored and where the mixing of polarizations is expected. Furthermore, the SCBA has an inherent limit value for $\epsilon^{2}$, defined by the threshold value beyond which the theory becomes unstable. The value of such an edge can be, however, lower than the one corresponding to the actual degree of elastic heterogeneity, e.g., observed by MD simulations in a Lennard-Jones glass [11]. The SCBA can successfully catch the trend and length scales related to the Rayleigh scattering $[5,11,19,22,42]$. The overall strength of the attenuation can be, however, underestimated $[10,11,38,42,43]$. Outcomes from this procedure are discussed in the Supplemental Material [88]. In terms of the perturbative series expansion, the SCBA includes some terms not accounted by the Born approximation or the GBA. We, however, observe that since the convergence properties of the perturbative series expansion are unknown to consider a larger number of terms, they do not necessarily improve the approximation.

We adopt the hypothesis of local isotropy and introduce the orthonormal basis defined by the direction of wave 
propagation (longitudinal) and the two orthogonal (transverse) ones [32]. On this basis, the "bare," the averaged Green's dyadic, and the self-energy are diagonal. The final expression exploited to describe the diagonal element of the mass operator in the GBA is

$$
\begin{aligned}
\Sigma_{k k}(\mathbf{q}, \omega)= & \hat{L}_{1 k k i i} \lim _{\eta \rightarrow 0^{+}} \frac{1}{{\tilde{c_{i}}}^{2}}\left\{\frac{1}{\tilde{q}_{0 i, \eta}^{2}-q^{2}}\right. \\
& \left.+\frac{1}{\left[\tilde{q}_{0 i, \eta}^{2}-q^{2}\right]^{2}} \frac{\epsilon^{2}}{\tilde{c}_{i}^{2}} q^{2} \Delta \tilde{\Sigma}_{i i}^{1}\left(0, \omega_{\eta}\right)\right\},
\end{aligned}
$$

where $\tilde{q}_{0 i, \eta}=\frac{\omega_{\eta}}{\tilde{c}_{i}}, \omega_{\eta}=\omega+i \eta, \eta$ is a positive real variable, $\Delta \tilde{\Sigma}_{i i}^{1}(\mathbf{q}, \omega)=\left(\epsilon^{2} q^{2}\right)^{-1}\left[\Sigma_{i i j j}^{1}(\mathbf{q}, \omega)-\Sigma_{i i j j}^{1}(0,0)\right], \tilde{c}_{i}=$ $\left[\left(c_{i}^{0}\right)^{2}+\epsilon^{2} \tilde{\Sigma}_{i i}^{1}(0,0)\right]^{1 / 2}, c_{i}^{0}$ is the phase velocity of the "bare" medium for polarization $i=L, T$. In the next paragraph we describe the passages leading from Eq. (5) to its approximate expression (7). Specifics of $\Sigma_{k k}(\mathbf{q}, \omega)$ computations are outlined in Appendix A. The repeated indices $k k, i i, j j=L, T$ with $L$ and $T$ labeling longitudinal and transverse directions, respectively. It becomes clear that the longitudinal and transverse self-energies are both composed by two terms, each one accounting, respectively, for the coupling with longitudinal and transverse dynamics, i.e., $\Sigma_{L(T)}=\Sigma_{L L(T T)}+\Sigma_{L T(T L)}$. We consider only spatial fluctuations of shear modulus in agreement with previous literature studies $[5,19,20]$. The introduction of fluctuations of the Lamé parameter $[22,31,32]$ is discussed in Supplemental Material [88]. Under the hypothesis of local isotropy, $\tilde{\mathbf{R}}(\mathbf{q})$ can be factorized in the product of a scalar correlation function $\tilde{r}(\mathbf{q})$ and a tensor, which depends only on the angle between the versors $\hat{q}$ and $\hat{s}$ [32]. We consider the simplest form of the shear modulus fluctuations' scalar correlation function, in real space an exponential decay with correlation length $a$, corresponding to randomly distributed spatial fluctuations. This function satisfies proper consistency requirements [35]. In the vectorial Fourier space it corresponds to the Lorentz function

$$
\tilde{r}(\mathbf{q})=\epsilon^{2} \frac{1}{\pi^{2}} \frac{q^{2} a^{-1}}{\left(q^{2}+a^{-2}\right)^{2}} .
$$

The integrations over the wave vector and the angular coordinates in Eq. (7) are performed analytically and numerically, respectively. The input parameters of the GBA are $a, \epsilon^{2}$, and $c_{L(T)}^{0}$.

Beyond disorder, local anisotropy in amorphous solids can promote or enforce the mixing of polarizations. It is possible, in the frame of RMT, to account for both local and statistical anisotropy [97]. We, however, neglected such effects here because our aim is to point out how, even in an isotropic medium, the disorder generates the mixing of polarizations.

\section{B. Developing the generalized Born approximation}

The GBA states for an approximate expression of the mass operator which includes corrective terms to the Born approximation in the framework of the perturbative series expansion [82]. The Born approximation corresponds to truncate the perturbative series expansion to the first order. The next order approximation is stated in Eq. (5), where the stochastic operator $\hat{\mathbf{L}}_{1}$ acts on $\left\langle G_{i i}(\mathbf{q}, \omega)\right\rangle^{1}=$ $\lim _{\eta \rightarrow 0^{+}} \frac{1}{\tilde{c}_{i}^{2}}\left\{\frac{1}{\tilde{q}_{0, \eta}^{2}-q^{2}-q^{2} \frac{\epsilon^{2}}{\tilde{c}_{i}^{2}} \Delta \tilde{\Sigma}_{i i}^{1}\left(\mathbf{q}, \omega_{\eta}\right)}\right\} . \quad\left\langle G_{i i}(\mathbf{q}, \omega)\right\rangle^{1}$ can be expanded in the Taylor series $\lim _{\eta \rightarrow 0^{+}} \sum_{n=0}^{\infty} \frac{\left[\frac{\epsilon_{i}^{2}}{\tilde{c}_{i}^{2}} q^{2} \Delta \tilde{\Sigma}_{i i}^{1}\left(\mathbf{q}, \omega_{\eta}\right)\right]^{n}}{\left[\tilde{q}_{0 i, \eta}^{2}-q^{2}\right]^{n+1}}$. The Taylor series is convergent almost everywhere in a domain of the $(q, \omega)$ plane where the conditions $\frac{\epsilon^{2}}{\tilde{c}_{i}^{2}}\left|\Delta \tilde{\Sigma}_{i i}^{1}(\mathbf{q}, \omega)\right|<1$ and $\operatorname{Im}\left[\Delta \tilde{\Sigma}_{i i}^{1}(\mathbf{q}, \omega)\right]>0$ are fulfilled [82]. In the case that the scalar correlation function is an exponential decay it is possible to find for each polarization a bounded region of the wave-vector-frequency plane $\Omega^{i}=\left(0, \omega_{\text {Max }}\right) \times\left(0, q_{\text {Max }}^{i}\right)$, where the conditions above are fulfilled and the Taylor series is thus almost everywhere convergent [82]. It follows that $\Sigma_{k k}(\mathbf{q}, \omega)$ in Eq. (5) can be approximated for frequencies and wave vectors inside the smaller of the $\Omega^{i}$ domains by the convergent series in the following [82]:

$$
\begin{aligned}
\Sigma_{k k}(\mathbf{q}, \omega) \sim & \lim _{\eta \rightarrow 0^{+}} \frac{1}{\tilde{c}_{i}^{2}} \sum_{n=0}^{\infty} \hat{\mathbf{L}}_{1 k k i i} \\
& \times\left\{\frac{\left[\frac{\epsilon^{2}}{\tilde{c}_{i}^{2}} q^{2} \Delta \tilde{\Sigma}_{i i}^{1}\left(\mathbf{q}, \omega_{\eta}\right)\right]^{n}}{\left[\tilde{q}_{0 i, \eta}^{2}-q^{2}\right]^{n+1}} \theta\left(q_{M a x}^{i}-q\right)\right\} .
\end{aligned}
$$

The function $\theta(q)$ is the Heaviside function. The domain of the wave-vector-frequency plane where Eq. (9) holds includes both the Rayleigh region and the wave-vector region $a q \sim$ 1 , where the mixing of polarizations is expected [82]. We truncate the Taylor series in Eq. (9) to the first order. Furthermore, up to wave vectors relevant to our interest, $q=O\left(a^{-1}\right)$, assuming $\Delta \tilde{\Sigma}^{1}(\mathbf{q}, \omega) \approx \Delta \tilde{\Sigma}^{1}(0, \omega)$ and then extending to infinity the upper integration boundary does not significatively enlarge the error related to the given approximation, while facilitating the analytical calculation [82]. Equation (7) is thus retrieved. We observe that, while $\Delta \tilde{\Sigma}_{i i}^{1}(\mathbf{q}, \omega)$ depends on the specific physical character of the wave fields and of the inhomogeneity domains, Eq. (9) preserves a generality character and can provide the basis for the implement of the GBA to fields other than acousticlike excitations in elastically heterogeneous media. Similarly, in place of a spatial random distribution of the heterogeneity domains leading to the exponential decay scalar correlation function, correlated disorder with the proper correlation function can in principle be considered.

\section{Analysis of theoretical outputs}

The experimental observations validate in glassy $[\mathrm{C} 8 \mathrm{MIM}] \mathrm{Cl}$ the presence of the bending of the characteristic energies dispersion (see Fig. 3). We superimpose to the dynamic structure factors calculated by GBA such a bending effect (see Fig. 6). This is obtained by a suitable normalization [98] of the frequency of $G^{0}(\mathbf{q}, \omega)$ entering in Eq. (3), i.e., $\tilde{\omega}=\omega\left[\frac{q_{0}}{q \pi} \sin \left(\frac{\pi q}{q_{0}}\right)\right]^{-1}$, where $\tilde{\omega}$ states for normalized and $\omega$ for unrenormalized frequency. In order to not overload the notation in the following the tilde will be omitted. $q_{0}=17.9 \mathrm{~nm}^{-1}$ is derived by the experimental dispersion of the characteristic energy of longitudinal excitations obtained by 1 -DHO fitting model (see Fig. 3). The possible broadening of the acousticlike excitations due to the existence of a distribution of nearest-neighbor values is also taken into 

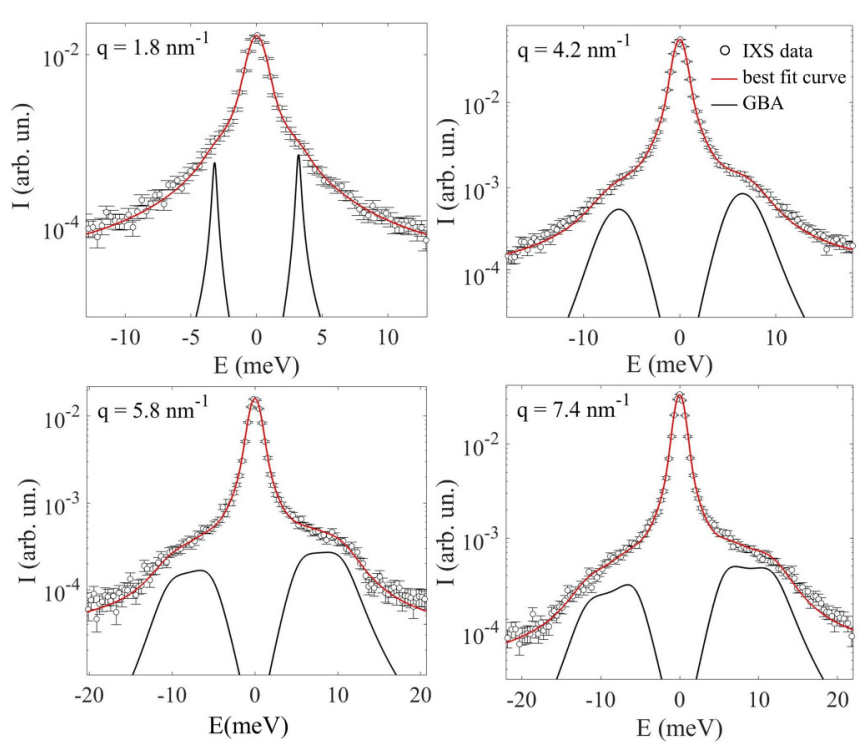

FIG. 4. Comparison between IXS spectra of glassy [C8MIM]Cl (black circles with error bars) and calculated spectra (red lines) obtained from GBA-modeled inelastic components (black lines) for selected values of $q$.

account, similarly to what is done in Ref. [54]. The detailed procedure is described in the Supplemental Material [88]. We, however, emphasize that the bending mostly influences the longitudinal dynamic structure factors at the edge or outside the first pseudo-Brillouin zone, leaving almost unchanged the spectra corresponding to wave vectors inside it (see Supplemental Material [88]).

The quantities characterizing $S_{L / T}(q, \omega)$ calculated by GBA, i.e., broadening $\Gamma_{L / T}(q)$, characteristic energy $\Omega_{L / T}(q)$, relative intensity $\tilde{I}_{L / T(2)}(q)$ can be derived by a so-called spectral function approach [23,31], i.e., by fitting the dynamic structure factors calculated by GBA with a fitting model composed by one or two DHO functions [Eq. (2)], as done for the IXS data. As was the case for experimental data of glassy [C8MIM]Cl, this protocol eases the comparison of GBA outcomes with results available in literature for different classes of glass, most of which have been obtained by exploiting the same phenomenological fitting model functions $[38,39,41-43,46,47,49-51]$. From $S_{L(T)}(q, E)$ obtained by GBA, $g(E) / E^{2}$ is derived by a numerical integration

$$
\frac{g(E)}{E^{2}}=\frac{2}{\pi q_{D}^{3}} \int_{0}^{q_{D}} d q\left[S_{L}(q, E)+2 S_{T}(q, E)\right],
$$

where $q_{D}$ is the Debye wave vector. The value of $q_{D}$ is obtained by the relationship $q_{D}=\left(6 \pi^{2}\right)^{\frac{1}{3}} \frac{q_{0}}{2 \pi}$.

\section{DISCUSSION}

The comparison between IXS spectra for selected $q$ values in the first pseudo-Brillouin zone and calculated spectra obtained by GBA is shown in Fig. 4. The dynamic structure factor $S_{L}(q, E)$ in Eq. (1) is modeled by exploiting the GBA in place of using the DHO-based model function in Eq. (2). A zero-centered delta function $I_{0} \delta(E)$ is, also in this (a)

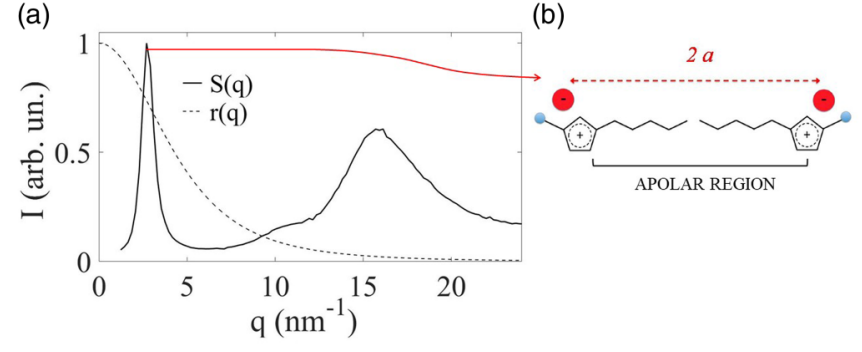

FIG. 5. (a) Measured $S(q)$ (black line) and scalar shear modulus fluctuation correlation function $\tilde{r}(\mathbf{q})$, used in GBA (dashed line). The correlation length $a$ is set to $\frac{\pi}{q_{\mathrm{FSDP}}}$. (b) Pictorial representation of the spatial configuration of glassy [C8MIM]Cl at nanoscale. The aggregation of the alkyl chain generates the nonpolar domains whose average size is $2 a \sim 2 \pi / q_{\mathrm{FSDP}}$.

case, added to $S_{L}(q, E)$. The agreement between experimental and theoretical spectra is very good. On an overall basis, the adjustable parameters of the GBA are $a, \epsilon^{2}, c_{L(T)}^{0}$ (see Sec. II). In the case of glassy [C8MIM] Cl, however, the input parameter $a$ of GBA is fixed to $\frac{\pi}{q_{\text {FSDP }}}$. Figure 5 shows the measured $S(q)$ and $\tilde{r}(q)$ with the assigned value of $a$. The underlying relationship between $q_{\mathrm{FSDP}}$ and $a$ is highlighted. The adjustable parameters of the GBA $\epsilon^{2}$ and $c_{L(T)}^{0}$ have been set by making the dispersion of $\Omega_{L}(q)$ and $\Gamma_{L}(q)$ obtained by fitting with 1-DHO or 2-DHO model functions the theoretical, GBA-based, $S_{L}(q, E)$ (see Sec. II C) overlapping the one derived from the experimental data with the same DHO-based fitting model function (Fig. 3). The numerical integration on the angular coordinate can indeed overly slow down the time of convergence of a direct fit of the experimental data with a fitting model function directly obtained by GBA. Remarkably, conversely to the case of DHO-based model function [Eq. (2)], the GBA-based model function can fit the experimental spectra in the whole first pseudo-Brillouin zone, without the need of introducing further adjustable parameters in the high- $q$ region, i.e., switching from 1-DHO to 2-DHO fitting model function. We notice, furthermore, that the input parameters of the GBA and of the Born approximation are the same. This allows to fully attribute the improvements introduced by GBA to the approximation scheme used and not to the introduction of further adjustable parameters. A comparison between the features of the longitudinal acousticlike excitations derived from GBA and Born approximation for the same input parameters is presented in the Supplemental Material [88]. It can be observed in Fig. 4 that for $q$ sufficiently large the GBA correctly describes the extra feature in the inelastic component observed in the IXS spectra (see Sec. I). As detailed below, the comparison with GBA results permits to unambiguously attribute such a feature to the mixing of polarizations. The $q$ trend of the extra feature observed in the GBA-based computation of $S_{L}(q, E)$ can be furthermore evaluated by inspection of Fig. 6 . The figure shows the projection on the $(q, E)$ plane of the dynamic structure factors and of the current spectra $C_{L}(q, E) \propto \frac{E^{2}}{q^{2}} S_{L}(q, E)$, obtained by GBA. Current spectra for two significant $q$ values are furthermore shown. The extra feature in $S_{L}(q, E)$ appears at $q$ high enough, while merging in the main peak when $q$ is lowered. 
(a)

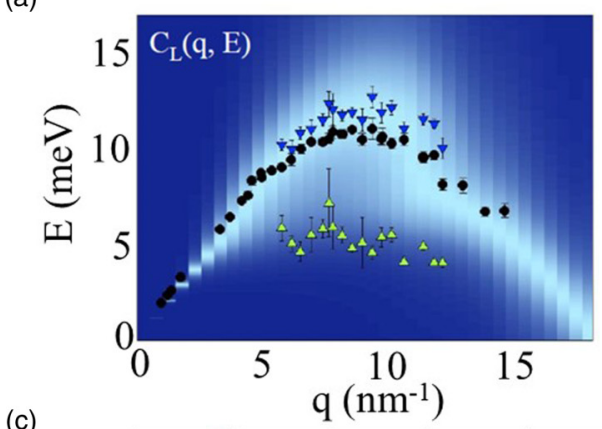

(c)

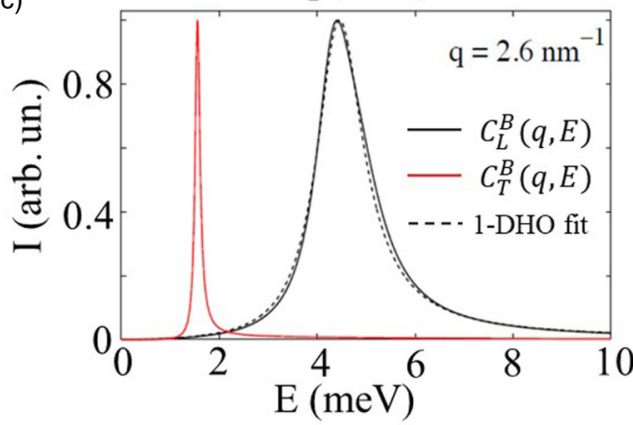

(b)

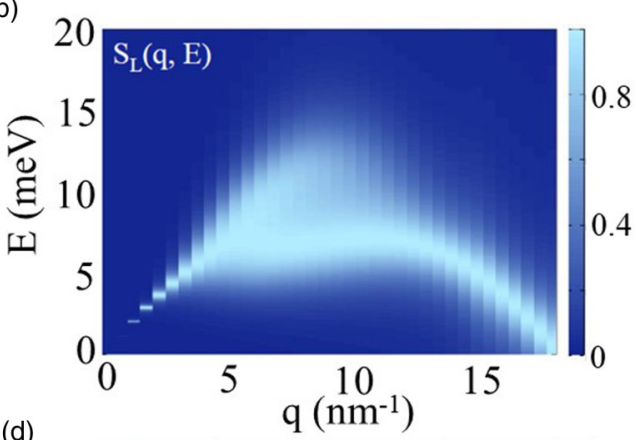

(d)

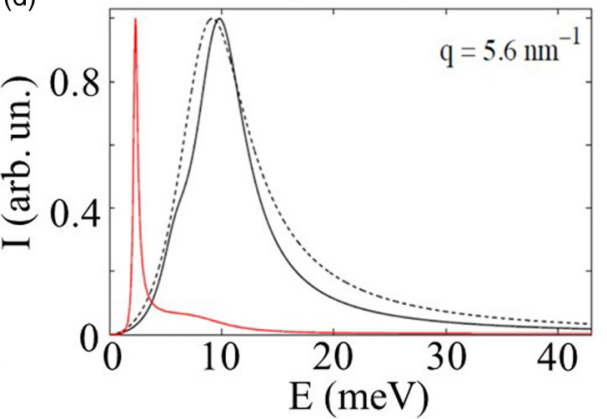

FIG. 6. (a) Projection on the $(q, E)$ plane of $C_{L}(q, E)$, obtained from GBA. The frequency normalization of the "bare" Green dyadic in Eq. (3) is enforced to account for the bending of the dispersion of the characteristic energies of acousticlike excitations. The maximum of $C_{L}(q, E)$ for each $q$ is normalized to one. Black circles show the experimental values of $\Omega_{L}$ obtained with the 1-DHO fitting model, blue and green triangles the values obtained with the 2-DHO fitting model. (b) Projection on the $(q, E)$ plane of $S_{L}(q, E)$. The low-energy feature related to the mixing of longitudinal and transverse polarizations is here highlighted. (c), (d) Representative $C_{L}(q, E)$ (black line) and $C_{T}(q, E)$ (red line) obtained by exploiting the GBA in the low- $q$ and high- $q$ regions. The low-energy shoulder in $C_{L}(q, E)$, generated by the mixing of polarizations, is clearly observable. The dashed line shows the best-fit curve of $C_{L}(q, E)$ obtained with 1-DHO fitting model.

It is closed to the characteristic frequency of the transverse excitation. The endorsement of the fact that such a secondary peak originates from the mixing of polarizations stems from the observation that it disappears when in the longitudinal self-energy $\Sigma_{L}=\Sigma_{L L}+\Sigma_{L T}$, the cross term accounting for the coupling with transverse dynamics $\Sigma_{L T}(q, \omega)$ is set to zero [82].

A detailed comparison between the experimentally and GBA-derived characteristic quantities of the longitudinal acoustic dynamics of [C8MIM]Cl glass is reported in Fig. 7. The phase velocity, corrected for the bending of the characteristic frequency dispersion $V_{L(T)}(q)$, shown in Fig. 7, is obtained through the relationship $V_{L(T)}(q)=$ $\Omega_{L(T)}(q) \frac{\pi}{q_{0} \sin \left(q \pi / q_{0}\right)}$. In the Rayleigh region $(a q \ll 1)$ the dynamic structure factor related to longitudinal acoustic dynamics is characterized by a well-defined inelastic excitation (see Figs. 4 and 6). The anomalous behavior in the Rayleigh region is properly reproduced: the phase velocity of the acousticlike excitations shows a gradual softening with respect to its macroscopic ( $q \rightarrow 0$ limit) value while the attenuation strongly increases, following the typical $q^{4}$ trend of the Rayleigh scattering [Figs. 7(a) and 7(c)]. We recall that Kramers-Kronig relations link the two quantities [5]. At the boundary of the Rayleigh region $(a q \lesssim 1)$ the phase velocity starts to increase and, consequently, a local minimum is observed in its trend. Meanwhile, a crossover from $q^{4}$ to $q^{2}$ power law is observed in the attenuation trend. Just below the transition to a $q^{2}$ trend, we observe a hump in the $\Gamma$ dispersion and a rapid increase of the phase velocity. These features are related to the coupling between longitudinal and transverse polarizations since they are also strongly affected by the removing of the cross term $\Sigma_{L T}$ in $\Sigma_{L}$. Similar features have been reported in a theoretical characterization of elastic excitations in polycrystalline aggregates drawn by the Born approximation and ascribed to the coupling of longitudinal with transverse dynamics [31]. The hump in the $\Gamma$ trend can be viewed as a prolongation of the $q^{4}$ behavior observed in the Rayleigh regime. It is stronger the bigger it is $\epsilon^{2}$ and as smaller $\frac{c_{T}^{0}}{c_{L}^{0}}$ (see also Supplemental Material [88]). This latter fact emphasizes how in the case of longitudinal acoustic dynamics at the edge of the Rayleigh region $(a q \lesssim 1)$, where depolarization effects begin to affect the acoustic dynamics, the coupling of polarizations, though not manifesting in a clear peaklike feature in the dynamic structure factors, contributes to the $\Gamma$ increase. A scalar model can thus underestimate the attenuation observed in this $q$ region. In the region $a q \sim 1$ the mixing of polarizations shows up, as discussed above. For $a q>1$ the coexistence of two excitations at different characteristic energies, finally, can be observed [31,33,99]. This feature can be reproduced also by using a scalar Born approximation $[31,33]$. In the case of glassy [C8MIM]Cl it is observable in the transverse dynamics, as discussed in the following.

The properties of the acoustic dynamics described above, observed in the experimental characterization of the glassy $[\mathrm{C} 8 \mathrm{MIM}] \mathrm{Cl}$ and retrieved by GBA, are typical of sev- 
(a)

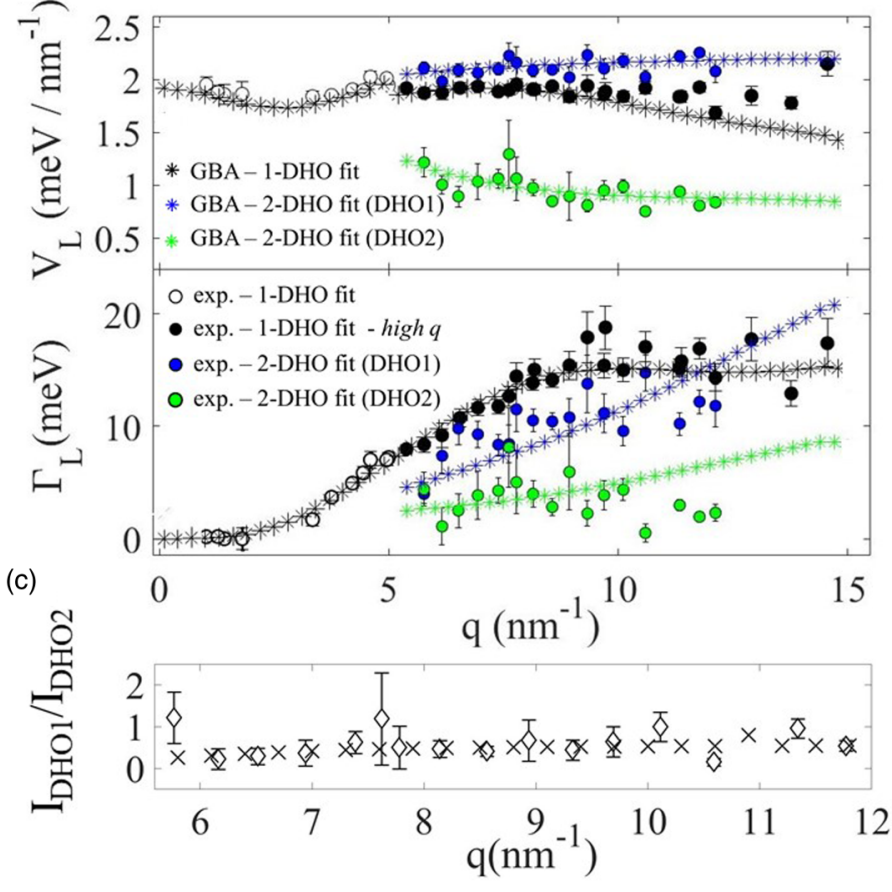

(b)
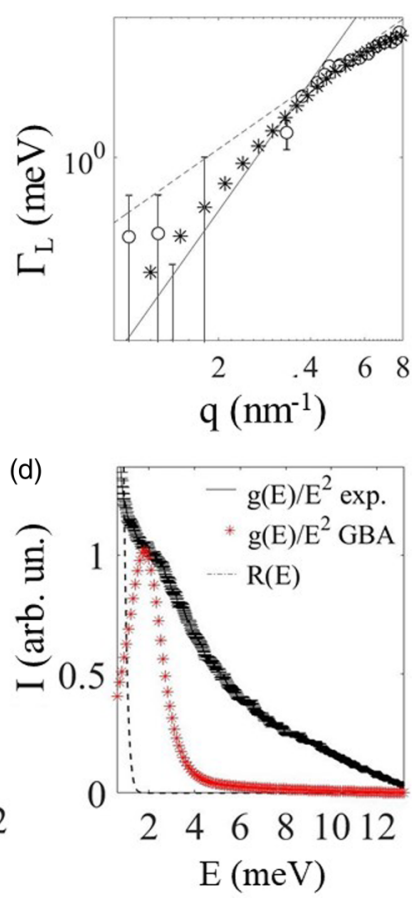

FIG. 7. (a) Phase velocity (upper graph) and broadening (lower graph) of longitudinal acousticlike excitations obtained by IXS and GBA as a function of $q$. Open and black circles show the values obtained from experimental data with the 1-DHO fitting model, respectively, in the low- and high- $q$ regions. Blue and green circles represent the values obtained with the 2-DHO fitting model. Stars mark the values derived from GBA. Black stars are obtained by exploiting 1-DHO fitting model, blue and green stars 2-DHO fitting model. (b) Experimental and theoretical broadening in the low- $q$ region. The presence of Rayleigh scattering is highlighted. Full and dashed lines are guides to eyes showing, respectively, the $q^{4}$ and $q^{2}$ trends. (c) Ratio of intensities of the two DHO functions modeling the inelastic features observed in the high- $q$ region observed in experimental data (diamonds) and GBA outcomes (crosses). (d) Experimentally derived $g(E) / E^{2}$ (black lines) and acousticlike contribution to $g(E) / E^{2}$ obtained from GBA (red stars). Dashed line represents the experimental energy resolution.

eral topologically disordered systems, as confirmed by the experimental studies reported in literature, mostly achieved by IXS or INS [22,39,41-43,46,47,49,51]. A quantitative agreement between experimental findings for [C8MIM]Cl glass and GBA outcomes is obtained in the whole first pseudo-Brillouin zone, as highlighted in Fig. 7. In particular, the following experimental features are quantitatively reproduced by the GBA: (i) the Rayleigh scattering causing the $q^{4}$ attenuation trend and the softening of the phase velocity; (ii) the crossover at $q_{c}$ from $q^{4}$ to $q^{2}$ trend in the $\Gamma_{L}(q)$ dispersion; (iii) the kink at $q_{c}$ in the $\Omega_{L}(q)$ dispersion; (iv) the presence of the low-energy shoulder in $S_{L}(q, E)$, related to the mixing of longitudinal and transverse polarization; (v) the relative intensity of the two inelastic features [Figs. 7(b) and 4]; (vi) the BP in the reduced VDOS. We stress that the GBA allows modeling only the acousticlike contribution to the VDOS and not the one arising from pure molecular modes. An accurate quantitative agreement is achieved, in particular the underestimation of the overall strength of attenuation, which affects other mean field RMTs, is prevented. The input parameters of the theory used to reproduce the experimental data are $c_{L}^{0}=2.29 \mathrm{meV} / \mathrm{nm}^{-1}$, $c_{T}^{0} / c_{L}^{0}=0.52$, which is a typical value for glasses [100], $\tilde{\epsilon}^{2}=\frac{\epsilon^{2}}{\bar{\mu}^{2}}=0.4$, where $\bar{\mu}$ is the average shear modulus, and $a=1.1 \mathrm{~nm}$. The value of $a$ is set by $S(q)$ measurements. The value of $c_{L}^{0}=3.48 \times 10^{3} \mathrm{~m} / \mathrm{s}$ is typical for glasses (see, e.g., Ref. [39]). There is any available measurements of speed of sound in glassy [C8MIM]Cl. This value is, however, consistent with the speed of sound value observed in liquid [C8MIM]Cl in the temperature range $T \in$ [280-340] K [101]. The value of $\tilde{\epsilon}^{2}$ can be compared with the one extrapolated from the numerical results of Ref. [60] for ILs with the same cation as $[\mathrm{C} 8 \mathrm{MIM}] \mathrm{Cl}$. By assuming that an estimation of $\tilde{\epsilon}^{2}$ can be obtained from the square of the ratio between the FWHM and the center of the Gaussian function describing the distribution of the values of local shear modulus in Ref. [60], the agreement is quite good. The Supplemental Material [88] shows how the features of GBA-based $S_{L}(q, E)$ transform by changing one at time the input parameters $a, \tilde{\epsilon}^{2}, c_{T}^{0} / c_{L}^{0}$.

Figures 8 and 9 finally show the features of the transverse dynamics obtained for the same input parameters used to reproduce the experimental results on longitudinal acoustic dynamics. In the low- $q$ region it is observed a well-defined inelastic excitation characterized by (i) a crossover from $q^{4}$ to $q^{2}$ trend in the $\Gamma_{T}$ dispersion at the $q$ point where the characteristic frequency of the acousticlike excitations matches the BP energy; (ii) a softening of the phase velocity in the Rayleigh regime; (iii) the Ioffe-Regel crossover at wave vector and frequency point where $\pi \Gamma_{T}$ becomes larger than $\Omega_{T}$, occurring near the BP energy. At larger $q$, a high-energy shoulder appears in $C_{T}(\mathbf{q}, \omega)$ (Figs. 6 and 8). This is not entirely related to the mixing of polarizations. It is indeed partially preserved when the term $\Sigma_{T L}$ is set to zero. These 
(a)

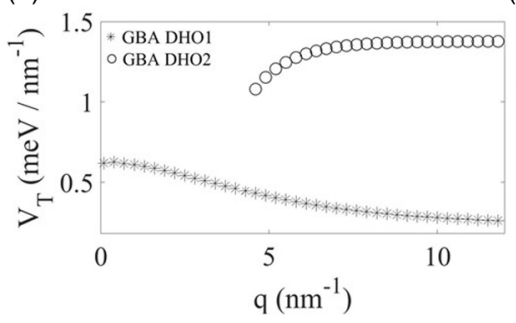

(b)

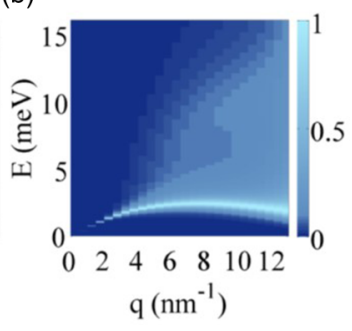

FIG. 8. Features of transverse dynamics obtained from GBA. (a) Phase velocities of the two inelastic excitations observed in $S_{T}(q, \omega)$. (b) Projection on the $(q, E)$ plane of $C_{T}(q, E)$.

results are in qualitative agreement with MD results in topological glasses $[2,5,40]$ or liquids at high wave vectors $[56,58]$. The characterization of the transverse dynamics that emerges from this study is further validated by the agreement between the characteristic energy of the BP predicted through GBA and observed by INS. The total VDOS is indeed mainly determined by transverse dynamics [5].

\section{CONCLUSION}

Within the framework of the RMT we developed a simple enough, mathematically tractable approximation allowing a unified description of the acoustic dynamics at nanoscale in glasses. The proposed model describes all the anomalies of acousticlike excitations characterizing an amorphous solid in the first pseudo-Brillouin zone, i.e., both the Rayleigh scattering and the mixing of longitudinal and transverse polarization observed at higher wave vectors in several topologically disordered systems. The fact that the mixing of polarizations is obtained as an outcome of an analytical model developed in the RMT framework for a model glass, i.e., a random medium with spatial fluctuating elastic moduli, in combination with the numerous observations in different classes of glass of features in $S(q, E)$, which can be traced back to it, outline how, similarly to the Rayleigh scattering, the mixing of polarizations can be treated as a general property of acousticlike excitations in amorphous solids. The insights which emerge
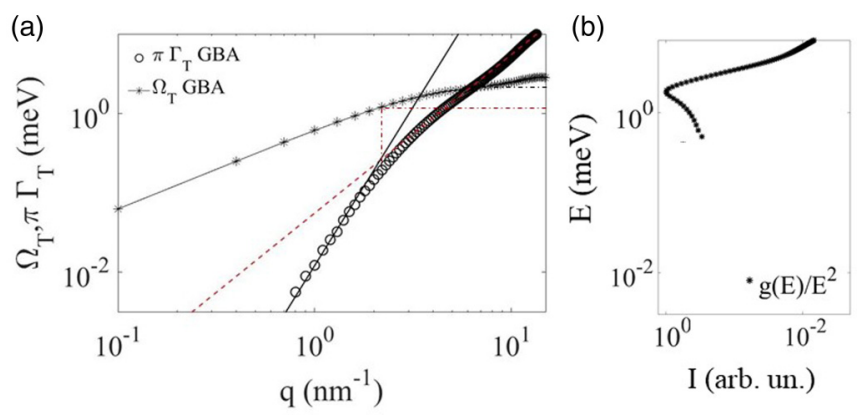

FIG. 9. Features of transverse dynamics obtained from GBA. (a) $\pi \Gamma_{T}$ (circles) and $\Omega_{T}$ (stars with line) of the low-frequency inelastic feature observed in $S_{T}(\mathbf{q}, \omega)$. Full black and red dashed lines are guides to eye showing, respectively, the $q^{4}$ and $q^{2}$ trends. The red and black dashed-dotted lines point out, respectively, the broadening crossover from $q^{4}$ to $q^{2}$ trend and the Ioffe-Regel crossover. (b) Reduced VDOS, $g(E) / E^{2}$. are in agreement with experimental and MD simulation observations reported in literature for different classes of amorphous solid. A quantitative agreement is, furthermore, observed for glassy [C8MIM]Cl, whose longitudinal acoustic dynamics and VDOS characterization, achieved by IXS and INS, respectively, has been reported in this paper. Significantly, the GBA allow a quantitative estimation of the acousticlike attenuation related to Rayleigh scattering avoiding underestimation, differently from other mean field models developed in the RMT framework.

The theoretical framework built up can be thought as a starting point for describing acoustic dynamics in different kinds of glasses, composites, artificially structured materials, ceramics, geophysical systems, or propagation of different kinds of waves in disordered media. An interesting area of application of the GBA may be the analysis of spin-wave propagation in heterogeneous antiferromagnetic or ferrimagnetic media. Although the polarization degrees of freedom are frozen in ferromagnets, they are fully unlocked in antiferromagnets or ferrimagnets [102]. Since recent developments of spintronics point to the design of magnetic logic gate based on polarized spin waves [103], a prospective application of GBA to heterogeneous magnetic media can have relevance even in the design of magnon devices. Under suitable modification of the scalar correlation function it is possible, furthermore, to include also systems characterized by correlated disorder, as well as quasiperiodically ordered metacrystals or phononic crystals [104]. It should be, however, emphasized that in all those cases where the size of the correlation length overlaps with the size of the pseudo-Brillouin zone, the proper bare Green dyadic, taking into account of the average periodicity and symmetry of the medium, has to be considered in order to calculate the self-energy, possibly involving the introduction of lattice Green's function formalism [70]. Another interesting case would be the study of acoustic properties in relaxor ferroelectrics, which are assumed to support polar regions of nanometric scale below their Burns temperature. The randomly distributed orientations of dipole moments may result in a heterogeneous spatial distribution of the elasticity tensor [105-107] and may thus be sensible to a study combining IXS and GBA. Finally, specific developments of this study can point toward a GBA solution of the Dyson equation related to a stochastic Navier-Stokes equation for a heterogeneous viscoelastic medium. This model is, e.g., introduced and discussed within the SCBA framework in Ref. [108]. A space- and frequency-dependent shear modulus is defined, encompassing a viscosity and high-frequency shear modulus varying in space, generalizing the Maxwellian space-independent viscoelastic coefficient. The exploiting of the GBA in this framework can be relevant because features in the dynamic structure factor possibly related to the mixing of polarizations have been observed experimentally $[50,54,109,110]$ and by MD simulations $[55,56,58]$ in several liquids. Similar developments could also support the understanding of the origin of the two-peak structure observed in the transverse current spectra of liquids in the high-frequency region, as attested by recent MD studies [57,111,112].

This study emphasizes also how the large tunability of the local structure of ionic liquids, which can be directed by controlling chemical and geometrical molecular structure, as 
well as the fact that their mesoscale local topology has been largely attested and analyzed in literature, can be exploited to test theoretical models.

\section{ACKNOWLEDGMENTS}

The authors thank W. Schirmacher, G. Pastore, and J. Dyre for useful discussions. M. Krisch and A. Bosak are acknowledged for support during the measurements at the ID28 beamline (ESRF), J. Taylor and R. Stuart during the measurements at ISIS (MARI spectrometer).

M.G.I. conceived and coordinated research. M.G.I. produced the theoretical results and the experimental proposals. A.M. introduced to the topic of ionic liquids. M.G.I., B.W., S.C., and A.G. did IXS measurements. M.G.I. and S.C. did INS measurements. M.G.I., B.W., and S.C. analyzed IXS data and discussed comparison with theoretical results. M.G.I. analyzed INS data. M.G.I., B.W., S.C., A.M., C.M., A.G., and G.R. discussed the results. M.G.I. wrote the paper. B.W., S.C., C.M., A.M., and G.R. revised the paper.

\section{APPENDIX A: SPECIFICS OF $\Sigma_{L(T)}(q, \omega)$ IN THE GENERALIZED BORN APPROXIMATION}

In the orthonormal basis defined by the direction of wave propagation $\hat{q}$ (longitudinal direction) and the two orthogonal ones (transverse directions) the averaged Green dyadic states

$$
\langle\mathbf{G}(\mathbf{q}, \omega)\rangle=\left\langle g_{L}(\mathbf{q}, \omega)\right\rangle \hat{q} \hat{q}+\left\langle g_{T}(\mathbf{q}, \omega)\right\rangle(I-\hat{q} \hat{q}) .
$$

Similar expressions can be retrieved for $G_{L(T)}^{0}(\mathbf{q}, \omega)$ and $\Sigma_{L(T)}(\mathbf{q}, \omega)$. As it follows from the properties of the inverse tensor, it is

$$
\left\langle g_{L(T)}(\mathbf{q}, \omega)\right\rangle=\frac{1}{g_{L(T)}^{0}(\mathbf{q}, \omega)^{-1}-\Sigma_{L(T)}(\mathbf{q}, \omega)} .
$$

The bare Green's functions are

$$
g_{L(T)}^{0}(\mathbf{q}, \omega)=\lim _{\eta \rightarrow 0^{+}} \frac{1}{\left(c_{L(T)}^{0}\right)^{2}} \frac{1}{\left(\frac{\omega+i \eta}{c_{L(T)}^{0}}\right)^{2}-q^{2}} .
$$

In the GBA each partial term of the self-energy, $\Sigma_{k k i i}(\mathbf{q}, \omega)$, is composed by two terms [see Eq. (7)], i.e.,

$$
\Sigma_{k k i i}(\mathbf{q}, \omega)=\Sigma_{k k i i}^{(0)}(\mathbf{q}, \omega)+\Sigma_{k k i i}^{(1)}(\mathbf{q}, \omega)
$$

with

$$
\begin{aligned}
& \Sigma_{k k i i}^{(0)}(\mathbf{q}, \omega)=\hat{L}_{1 k k i i} \lim _{\eta \rightarrow 0^{+}} \frac{1}{\tilde{c}_{i}^{2}} \frac{1}{\tilde{q}_{0 i, \eta}^{2}-q^{2}}, \\
& \Sigma_{k k i i}^{(1)}(\mathbf{q}, \omega)=\hat{L}_{1 k k i i} \lim _{\eta \rightarrow 0^{+}} \frac{1}{\tilde{c}_{i}^{2}} \frac{\frac{\epsilon^{2}}{\tilde{c}_{i}^{2}} q^{2} \Delta \tilde{\Sigma}_{i i}^{1}\left(0, \omega_{\eta}\right)}{\left[\tilde{q}_{0 i, \eta}^{2}-q^{2}\right]^{2}} \text {. }
\end{aligned}
$$

We define $\tilde{q}_{0 i}=\frac{\omega}{\tilde{c}_{i}}$, redefine for sake of simplicity $\frac{\eta}{\tilde{c}_{i}}=\eta$, and use the explicit expression of the operator $\hat{\mathbf{L}}_{1}$ stated in Eq. (2). Under the local isotropy hypothesis, in the orthonormal basis defined above, it is [32]

$$
\begin{aligned}
& \Sigma_{L L}^{(0)}(\mathbf{q}, \omega)=\lim _{\eta \rightarrow 0^{+}} \int \hat{q} \hat{q} \hat{s} \hat{s} d^{3} s \tilde{r}(\mathbf{q}-\mathbf{s}) \frac{1}{\tilde{c}_{L}^{2}} \frac{1}{\left(\tilde{q}_{0 L}+i \eta\right)^{2}-q^{2}}, \\
& \Sigma_{L L}^{(1)}(\mathbf{q}, \omega)=\lim _{\eta \rightarrow 0^{+}} \int \hat{q} \hat{q} \hat{s} \hat{s} d^{3} s \tilde{r}(\mathbf{q}-\mathbf{s}) \frac{1}{\tilde{c}_{L}^{2}} \frac{\frac{\epsilon^{2}}{\tilde{L}_{L}^{2}} s^{2} \Delta \tilde{\Sigma}_{L}^{1}(0, \omega+i \eta)}{\left[\left(\tilde{q}_{0 L}+i \eta\right)^{2}-q^{2}\right]^{2}}, \\
& \Sigma_{L T}^{(0)}(\mathbf{q}, \omega)=\lim _{\eta \rightarrow 0^{+}} \int \hat{q} \hat{q}(\mathbf{I}-\hat{s} \hat{s}) d^{3} s \tilde{r}(\mathbf{q}-\mathbf{s}) \frac{1}{\tilde{c}_{T}^{2}} \frac{1}{\left(\tilde{q}_{0 T}+i \eta\right)^{2}-q^{2}}, \\
& \Sigma_{L T}^{(1)}(\mathbf{q}, \omega)=\lim _{\eta \rightarrow 0^{+}} \int \hat{q} \hat{q}(\mathbf{I}-\hat{s} \hat{s}) d^{3} s \tilde{r}(\mathbf{q}-\mathbf{s}) \frac{1}{\tilde{c}_{T}^{2}} \frac{\frac{\epsilon_{T}^{2}}{\tilde{\tilde{c}}_{T}^{2}} s^{2} \Delta \tilde{\Sigma}_{T}^{1}(0, \omega+i \eta)}{\left[\left(\tilde{q}_{0 T}+i \eta\right)^{2}-q^{2}\right]^{2}}, \\
& \Sigma_{T T}^{(0)}(\mathbf{q}, \omega)=\frac{1}{2} \lim _{\eta \rightarrow 0^{+}} \int(\mathbf{I}-\hat{q} \hat{q})(\mathbf{I}-\hat{s} \hat{s}) d^{3} s \tilde{r}(\mathbf{q}-\mathbf{s}) \frac{1}{\tilde{c}_{T}^{2}} \frac{1}{\left(\tilde{q}_{0 T}+i \eta\right)^{2}-q^{2}}, \\
& \Sigma_{T T}^{(1)}(\mathbf{q}, \omega)=\frac{1}{2} \lim _{\eta \rightarrow 0^{+}} \int(\mathbf{I}-\hat{q} \hat{q})(\mathbf{I}-\hat{s} \hat{s}) d^{3} s \tilde{r}(\mathbf{q}-\mathbf{s}) \frac{1}{\tilde{c}_{T}^{2}} \frac{\frac{\epsilon^{2}}{\tilde{c}_{T}^{2}} s^{2} \Delta \tilde{\Sigma}_{T}^{1}(0, \omega+i \eta)}{\left[\left(\tilde{q}_{0 T}+i \eta\right)^{2}-q^{2}\right]^{2}}, \\
& \Sigma_{T L}^{(0)}(\mathbf{q}, \omega)=\frac{1}{2} \lim _{\eta \rightarrow 0^{+}} \int(\mathbf{I}-\hat{q} \hat{q}) \hat{s} \hat{s} d^{3} s \tilde{r}(\mathbf{q}-\mathbf{s}) \frac{1}{\tilde{c}_{L}^{2}} \frac{1}{\left(\tilde{q}_{0 L}+i \eta\right)^{2}-q^{2}}, \\
& \Sigma_{T L}^{(1)}(\mathbf{q}, \omega)=\frac{1}{2} \lim _{\eta \rightarrow 0^{+}} \int(\mathbf{I}-\hat{q} \hat{q}) \hat{s} \hat{s} d^{3} s \tilde{r}(\mathbf{q}-\mathbf{s}) \frac{1}{\tilde{c}_{L}^{2}} \frac{\frac{\epsilon^{2}}{\tilde{c}_{L}^{2}} s^{2} \Delta \tilde{\Sigma}_{L}^{1}(0, \omega+i \eta)}{\left[\left(\tilde{q}_{0 L}+i \eta\right)^{2}-q^{2}\right]^{2}} .
\end{aligned}
$$

We perform the tensor products in spherical coordinates in Eqs. (A1)-(A4). The tensor product results are the same of the ones reported in Refs. [31,32]. Therein, however, the arguments of the $|\mathbf{s}|$ integrals are different. We obtain

$$
\begin{aligned}
& \Sigma_{L L(L T)}^{(0)}(\mathbf{q}, \omega)=\epsilon^{2} q^{2} \int_{-1}^{+1} d x L_{L L(L T)}^{\mu \mu}(x) \frac{1}{\tilde{c}_{L(T)}^{2}} \frac{2}{\pi} a^{-1} I_{L(T)}^{(0)}(\mathbf{q}, \omega, x) \\
& \Sigma_{L L(L T)}^{(1)}(\mathbf{q}, \omega)=\epsilon^{2} q^{2} \int_{-1}^{+1} d x L_{L L(L T)}^{\mu \mu}(x) \frac{1}{\tilde{c}_{L(T)}^{2}} \frac{2}{\pi} a^{-1} I_{L(T)}^{(1)}(\mathbf{q}, \omega, x) \frac{\epsilon^{2}}{\tilde{c}_{L(T)}^{2}} \Delta \tilde{\Sigma}_{L(T)}^{1}(0, \omega),
\end{aligned}
$$




$$
\begin{aligned}
& \Sigma_{T T(T L)}^{(0)}(\mathbf{q}, \omega)=\epsilon^{2} q^{2} \frac{1}{2} \int_{-1}^{+1} d x L_{T T(T L)}^{\mu \mu}(x) \frac{1}{\tilde{c}_{T(L)}^{2}} \frac{2}{\pi} a^{-1} I_{T(L)}^{(0)}(\mathbf{q}, \omega, x), \\
& \Sigma_{T T(T L)}^{(1)}(\mathbf{q}, \omega)=\epsilon^{2} q^{2} \frac{1}{2} \int_{-1}^{+1} d x L_{T T(T L)}^{\mu \mu}(x) \frac{1}{\tilde{c}_{T(L)}^{2}} \frac{2}{\pi} a^{-1} I_{T(L)}^{(1)}(\mathbf{q}, \omega, x) \frac{\epsilon^{2}}{\tilde{c}_{T(L)}^{2}} \Delta \tilde{\Sigma}_{T(L)}^{1}(0, \omega),
\end{aligned}
$$

where $x=\cos (\hat{q} s)$, being $\hat{q} s$ the angle between the two versors $\hat{q}$ and $\hat{s}$, and $L_{L L}^{\mu \mu}(x)=4 x^{4}, L_{L T(T L)}^{\mu \mu}=4\left(1-x^{2}\right) x^{2}, L_{T T}^{\mu \mu}=$ $1-3 x^{2}+4 x^{4}$. The transverse degeneracy is, furthermore, taken into account. The functions $I_{k}^{(n)}(\mathbf{q}, \omega, x)$ with $k=L, T$ and $n=0,1$, appearing in Eqs. (A5) and (A6), are defined by the following integrals:

$$
I_{k}^{(n)}(\mathbf{q}, \omega, x)=\lim _{\eta \rightarrow 0^{+}} \int_{0}^{\infty} d s s^{2} \frac{s^{2}}{\left[a^{-2}+q^{2}+s^{2}-2 q s x\right]^{2}} \frac{\left[s^{2}\right]^{n}}{\left[\left(\tilde{q}_{0 k}+i \eta\right)^{2}-s^{2}\right]^{n+1}} .
$$

The integrals in Eq. (A7) can be calculated by exploiting a generalization of the Sokhotski-Plemelj theorem due to Fox [113,114]. The Sokhotski-Plemelj-Fox theorem states that

$$
\lim _{\eta \rightarrow 0^{ \pm}} \int_{a}^{b} d r \frac{f(r)}{\left[r-\left(r_{0} \mp i \eta\right)\right]^{n+1}}=\# \int_{a}^{b} d r \frac{f(r)}{\left[r-r_{0}\right]^{n+1}} \pm\left.\frac{i \pi}{n !} \frac{d^{n}}{d z^{n}} f(z)\right|_{z=r_{0}},
$$

where $a, b, r_{0}$, and $r$ are real variables: $a<r_{0}<b, f(r)$ is a function which admits a complex extension $f(z)$, which is analytical in a region of the complex plane containing the interval $[a, b]$ but not $r_{0}$. The symbol \# states for the Hadamard finite part integral, equal to the Cauchy principal value when $n=0$. We recognize in the second term of the second side of Eq. (A8) the residue of order $n$ of the integrand around the pole $r_{0}$, $\operatorname{Res}^{n}\left(r_{0}\right)=\left.\frac{1}{n !} \frac{d^{n}}{d z^{n}} f(z)\right|_{z=r_{0}}$. Relying on Eq. (A8) simple passages lead to

$$
\begin{aligned}
I_{k}^{(n)}(\mathbf{q}, \omega, x)= & \lim _{\eta \rightarrow 0^{+}} \int_{0}^{\infty} d s s^{2} \frac{s^{2}}{\left[a^{-2}+q^{2}+s^{2}-2 q s x\right]^{2}} \frac{\left[s^{2}\right]^{n}}{\left[\left(\tilde{q}_{0 k}+i \eta\right)^{2}-s^{2}\right]^{n+1}}=\# \int_{0}^{\infty} d s s^{2} \frac{s^{2}}{\left[a^{-2}+q^{2}+s^{2}-2 q s x\right]^{2}} \\
& \times \frac{\left[s^{2}\right]^{n}}{\left[\tilde{q}_{0 k}^{2}-s^{2}\right]^{n+1}}+\left.(-1)^{n+1} \frac{i \pi}{n !} \frac{d^{n}}{d z^{n}}\left\{z^{2} \frac{z^{2}}{\left[a^{-2}+q^{2}+z^{2}-2 q z x\right]^{2}} \frac{\left[z^{2}\right]^{n}}{\left[\tilde{q}_{0 k}+z\right]^{n+1}}\right\}\right|_{z=\tilde{q}_{0 k} .}
\end{aligned}
$$

The second term in the second side of Eq. (A9) can be immediately calculated. Considering the first term, we notice that by using integration by parts the Hadamard finite part integral can be traced back to a Cauchy principal value:

$$
\begin{aligned}
\# & \int_{0}^{\infty} d s s^{2} \frac{s^{2}}{\left[a^{-2}+q^{2}+s^{2}-2 q s x\right]^{2}} \frac{\left[s^{2}\right]^{n}}{\left[\tilde{q}_{0}^{2}-s^{2}\right]^{n+1}} \\
& =\text { P.V. } \int_{0}^{\infty} d s \frac{1}{n !}(-1)^{n+1} \frac{1}{\left(\tilde{q}_{0}-s\right)} \frac{d^{n}}{d s^{n}}\left\{s^{2} \frac{s^{2}}{\left[a^{-2}+q^{2}+s^{2}-2 q s x\right]^{2}} \frac{\left[s^{2}\right]^{n}}{\left[\tilde{q}_{0}+s\right]^{n+1}}\right\} .
\end{aligned}
$$

The Hadamard finite part integral thus exists because it exists the Cauchy principal value of the integral in the second side of Eq. (A10) since the integrand satisfies the Lipschitz property. The Cauchy principal value of the integral in Eq. (A10) can be calculated by exploiting the residue theorem because in the path of the complex plane where the Cauchy principal value is defined, which excludes the pole in $\tilde{q}_{0}$, the integrand has only nonessential singularities. In order to achieve a complete evaluation of the integrals in Eqs. (A1)-(A6), the quantities $\Delta \tilde{\Sigma}_{L(T)}^{1}(0, \omega)$ need yet to be characterized. $\Delta \tilde{\Sigma}_{L(T)}^{1}(\mathbf{q}, \omega)=\tilde{\Sigma}_{L(T)}^{1}(\mathbf{q}, \omega)-$ $\tilde{\Sigma}_{L(T)}^{1}(0,0)$, where $\tilde{\Sigma}_{L(T)}$ is related to the first-step self-energy obtained through the Born approximation $\Sigma_{L(T)}^{1}(\mathbf{q}, \omega)$ being

$$
\begin{gathered}
\Sigma_{L}^{1}(\mathbf{q}, \omega)=\epsilon^{2} q^{2} \tilde{\Sigma}_{L}^{1}(\mathbf{q}, \omega)=\epsilon^{2} q^{2}\left[\tilde{\Sigma}_{L L}^{1}(\mathbf{q}, \omega)+\tilde{\Sigma}_{L T}^{1}(\mathbf{q}, \omega)\right]=\int \hat{q} \hat{q} \tilde{r}(\mathbf{q}-\mathbf{s})\left[g_{L}^{0}(s, \omega) \hat{s} \hat{s}+g_{T}^{0}(s, \omega)(\mathbf{I}-\hat{s} \hat{s})\right] d^{3} s, \\
\Sigma_{T}^{1}(\mathbf{q}, \omega)=\epsilon^{2} q^{2} \tilde{\Sigma}_{T}^{1}(\mathbf{q}, \omega)=\epsilon^{2} q^{2}\left[\tilde{\Sigma}_{T T}^{1}(\mathbf{q}, \omega)+\tilde{\Sigma}_{T L}^{1}(\mathbf{q}, \omega)\right]=\frac{1}{2} \int(\mathbf{I}-\hat{q} \hat{q}) \tilde{r}(\mathbf{q}-\mathbf{s})\left[g_{L}^{0}(s, \omega) \hat{s} \hat{s}+g_{T}^{0}(s, \omega)(I-\hat{s} \hat{s})\right] d^{3} s .
\end{gathered}
$$

By comparing Eqs. (A11) and (A12) with Eqs. (A1)-(A4) it is immediate to verify that $\Sigma_{L(T)}^{1}$ is equivalent to $\Sigma_{L(T)}^{(0)}$ under the substitution $\tilde{c}_{L(T)} \rightarrow c_{L(T)}^{0}$.

\section{APPENDIX B: COUPLING OF ACOUSTICLIKE EXCITATIONS WITH INTERMOLECULAR VIBRATIONAL MODES}

The reduced VDOS of [C8MIM]Cl glass in the energy region $[7,14] \mathrm{meV}$ is characterized by a broad peaklike feature, related to the presence of IVMs, which have characteristic frequencies inside this energy interval as attested by Raman spectroscopy [89]. An IVM can couple to acousticlike excitations, affecting their effective phase velocity and attenuation when its characteristic frequency matches the one of the acousticlike excitations $[115,116]$. 
(a)

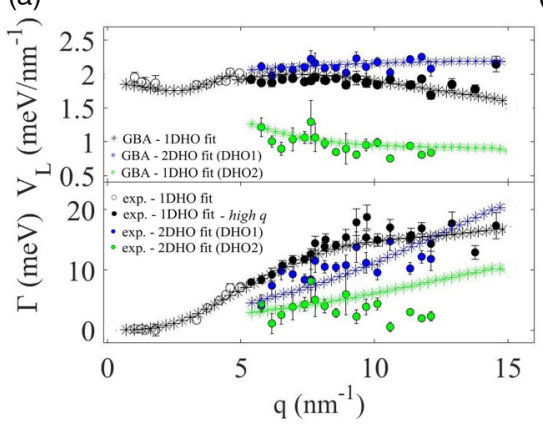

(b)

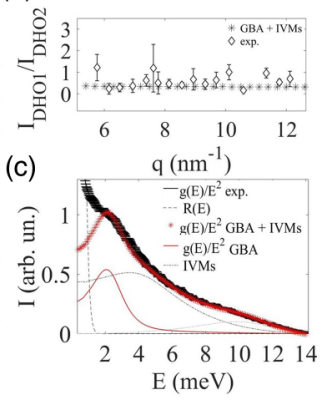

FIG. 10. (a) Phase velocity (upper graph) and broadening (lower graph) as a function of $q$ of the longitudinal acousticlike excitations obtained by IXS data (circles) and GBA-derived acousticlike excitations coupled to IVMs (stars). Open and black circles show experimental outcomes obtained with the 1-DHO fitting model, respectively, in the low- and high- $q$ regions, blue and green circles display the values obtained with the 2-DHO fitting model. Stars mark the values derived from theoretical outcomes, black stars show the results obtained with the 1-DHO fitting model, blue and green stars with the 2-DHO fitting model. (b) Ratio of intensities of the DHO functions related to the two inelastic features in the high- $q$ region. (c) Experimental (black lines) and theoretical (red stars) reduced VDOS, $g(E) / E^{2}$. Red and dotted-dashed lines show, respectively, the components of the reduced VDOS related to acoustic dynamics and IVMs. Dashed line shows the experimental INS resolution.

To account for the coupling between acousticlike excitations, described in the GBA framework, and IVMs we introduce an additional term $\Sigma_{L(T)}^{\mathrm{IVM}}$ to the self-energy calculated by GBA, $\Sigma_{L(T)}$. The coupling of the IVM with acousticlike excitations is treated to lowest order [3]. It is thus $\Sigma_{L(T)}^{\mathrm{IVM}}=\frac{q^{2} A_{L(T)}^{s}}{\omega^{2}-\omega_{0}^{2}+i \omega \Gamma_{\Gamma}}$, where $\omega_{0}$ is the characteristic frequency of the IVM, $\Gamma_{s}$ the broadening, and $A_{L(T)}^{s}$ is a polarization-dependent coupling factor. The total self-energy $\Sigma_{L(T)}^{t}=\Sigma_{L(T)}+\sum_{n}\left[\Sigma_{L(T)}^{\mathrm{IVM}_{n}}\right]$ enters into the expression of $\left\langle G_{L, T}(\mathbf{q}, \omega)\right\rangle$ through Eq. (1) in the text. $n$ indexes IVMs. We furthermore introduce the IVM's dynamic structure factor and define the corresponding IVM's contribution to the reduced

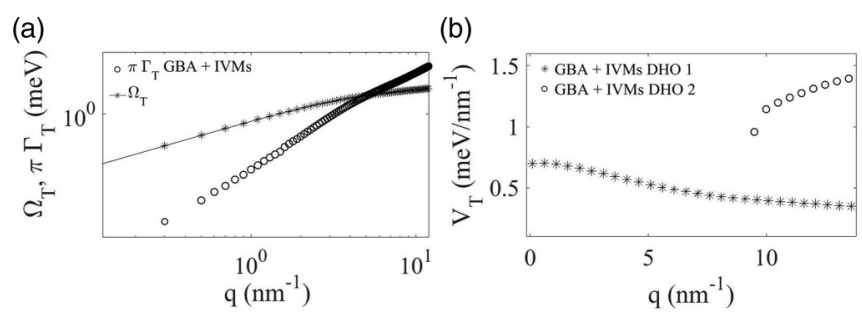

FIG. 11. (a) $\Gamma_{T}$, multiplied by $\pi$ (circles) and $\Omega_{T}$ (stars with line), of the low-frequency inelastic feature observed in $S_{T}(\mathbf{q}, \omega)$. (b) Phase velocity of the two features of the inelastic components observed in $S_{T}(\mathbf{q}, \omega)$.

VDOS:

$$
\begin{aligned}
S^{\mathrm{IVM}}(\mathbf{q}, \omega)= & \frac{1}{\pi} \frac{q^{2}}{\omega} \operatorname{Im}\left\{\left[\omega^{2}-\omega_{0}^{2}+i \omega \Gamma_{s}+q^{2}\left(A_{L}^{s}\right.\right.\right. \\
& \left.\left.\left.\times\left\langle g_{L}(\mathbf{q}, \omega)\right\rangle+2 A_{T}^{s}\left\langle g_{T}(\mathbf{q}, \omega)\right\rangle\right)\right]^{-1}\right\}, \\
\frac{g^{\mathrm{IVM}}(E)}{E^{2}}= & \frac{2}{\pi q_{D}^{3}} \int_{0}^{q_{D}} d q S^{\mathrm{IVM}}(\mathbf{q}, \omega) .
\end{aligned}
$$

The contribution of IVMs to the reduced VDOS, $\sum_{n} \frac{g^{\mathrm{IVM}}(E)}{E^{2}}$, is added to that related to the acoustic dynamics to obtain $\frac{g(E)}{E^{2}}$. To cope with literature data [89] we introduce two IVMs with, respectively, characteristic frequency and attenuation $\omega_{0}^{(1)}=7.2 \mathrm{meV}, \Gamma_{s}^{(1)}=7.5 \mathrm{meV}$ and $\omega_{0}^{(2)}=11 \mathrm{meV}, \Gamma_{s}^{(2)}=$ $6.5 \mathrm{meV}$. The value of the parameters $A_{L(T)}^{s(1,2)}$ has been set in order to reproduce the measured VDOS. Outcomes from this modeling are shown in Fig. 10 for the longitudinal dynamics. They are contrasted against the corresponding experimental features. The input parameters of the GBA are the same when the coupling parameter is set to zero, but $c_{T}^{0} / c_{L}^{0}$ is moved from 0.52 to 0.54 . Figure 11 shows the outcomes for the transverse dynamics. The presence of IVMs can influence the broadening of $S_{L(T)}(\mathbf{q}, \omega)$ at frequencies higher than the BP frequency, as well as slightly shifting the position of the BP in the reduced VDOS. It does not account for the mixing of polarization of acousticlike excitations, which can be instead described only by GBA.
[1] W. A. Phillips, Amorphous Solids: Low Temperature Properties (Springer, Berlin, 1981).

[2] H. Shintani and H. Tanaka, Nat. Mater. 7, 870 (2008).

[3] E. Maurer and W. Schirmacher, J. Low Temp. Phys. 137, 453 (2004).

[4] U. Buchenau, Y. M. Galperin, V. L. Gurevich, and H. R. Schober, Phys. Rev. B 43, 5039 (1991).

[5] A. Marruzzo, W. Schirmacher, A. Fratalocchi, and G. Ruocco, Sci. Rep. 3, 1407 (2013).

[6] A. I. Chumakov, G. Monaco, A. Monaco, W. A. Crichton, A. Bosak, R. Ruffer, A. Meyer, F. Kargl, L. Comez, D. Fioretto, H. Giefers, S. Roitsch, G. Wortmann, M. H. Manghnani, A. Hushur, Q. Williams, J. Balogh, K. Parlinski, P. Jochym, and P. Piekarz, Phys. Rev. Lett. 106, 225501 (2011).

[7] A. I. Chumakov, G. Monaco, A. Fontana, A. Bosak, R. P. Hermann, D. Bessas, B. Wehinger, W. A. Crichton, M. Krisch,
R. Ruffer, G. Baldi, G. Carini, G. Carini, G. DAngelo, E. Gilioli, G. Tripodo, M. Zanatta, B. Winkler, V. Milman, K. Refson et al., Phys. Rev. Lett. 112, 025502 (2014).

[8] V. L. Gurevich, D. A. Parshin, and H. R. Schober, Phys. Rev. B 67, 094203 (2003).

[9] D. A. Parshin, H. R. Schober, and V. L. Gurevich, Phys. Rev. B 76, 064206 (2007).

[10] H. Mizuno and A. Ikeda, Phys. Rev. E 98, 062612 (2018).

[11] H. Mizuno and S. Mossa, Condens. Matter Phys. 22, 43604 (2019).

[12] H. Mizuno, H. Shiba, and A. Ikeda, Proc. Natl. Acad. Sci. USA 114, E9767 (2017).

[13] H. Mizuno, S. Mossa, and J.-L. Barrat, Phys. Rev. B 94, 144303 (2016).

[14] H. Mizuno, S. Mossa, and J.-L. Barrat, Proc. Natl. Acad. Sci. USA 111, 11949 (2014). 
[15] L. Wang, A. Ninarello, P. Guan, L. Berthier, G. Szamel, and E. Flenner, Nat. Commun. 10, 26 (2019).

[16] L. Angelani, M. Paoluzzi, G. Parisi, and G. Ruocco, Proc. Natl. Acad. Sci. USA 115, 8700 (2018).

[17] E. Lerner, G. During, and E. Bouchbinder, Phys. Rev. Lett. 117, 035501 (2016).

[18] G. Kapteijns, E. Bouchbinder, and E. Lerner, Phys. Rev. Lett. 121, 055501 (2018).

[19] W. Schirmacher, G. Ruocco, and T. Scopigno, Phys. Rev. Lett. 98, 025501 (2007).

[20] W. Schirmacher, B. Schmid, C. Tomaras, G. Viliani, G. Baldi, G. Ruocco, and T. Scopigno, Phys. Stat. Sol. C 5, 862 (2008).

[21] S. Kohler, G. Ruocco, and W. Schirmacher, Phys. Rev. B 88, 064203 (2013).

[22] C. Ferrante, E. Pontecorvo, G. Cerullo, A. Chiasera, G. Ruocco, W. Schirmacher, and T. Scopigno, Nat. Commun. 4, 1793 (2013).

[23] P. Sheng, Introduction To Wave Scattering, Localization and Mesoscopic Phenomena (Academic, San Diego, 1995).

[24] C. S. O'Hern, L. E. Silbert, A. J. Liu, and S. R. Nagel, Phys. Rev. E 68, 011306 (2003).

[25] J. M. Carpenter and C. A. Pellizzari, Phys. Rev. B 12, 2391 (1975).

[26] Y. M. Beltukov, V. I. Kozub, and D. A. Parshin, Phys. Rev. B 87, 134203 (2013).

[27] M. C. C. Ribeiro, M. Wilson, and P. A. Madden, J. Chem. Phys. 108, 9027 (1998).

[28] U. Buchenau, N. Nucker, and A. J. Dianoux, Phys. Rev. Lett. 53, 2316 (1984).

[29] U. Buchenau, M. Prager, N. Nucker, A. J. Dianoux, N. Ahmad, and W. A. Phillips, Phys. Rev. B 34, 5665 (1986).

[30] S. Gelin, H. Tanaka, and A. Lemaitre, Nat. Mater. 15, 1177 (2016).

[31] M. Calvet and L. Margerin, J. Acoust. Soc. Am. 131, 1843 (2012).

[32] A. J. Turner and P. Anugonda, J. Acoust. Soc. Am. 109, 1787 (2001).

[33] S. M. Rytov, Y. A. Kravtsov, and V. I. Tatarskii, Principles of Statistical Radiophysics 4-Wave Propagation Trough Random Media (Springer, Berlin, 1989).

[34] K. Sobczyk, Stochastic Wave Propagation (Elsevier, Warszawa, 1985).

[35] S. Torquato, J. Chem. Phys. 111, 8832 (1999).

[36] W. Schirmacher, V. Folli, C. Ganter, and G. Ruocco, J. Phys. A: Math. Theor. 52, 464002 (2019).

[37] M. Mezard, G. Parisi, and A. Zee, Nucl. Phys. B 559, 689 (1999).

[38] B. Rufflé, D. A. Parshin, E. Courtens, and R. Vacher, Phys. Rev. Lett. 100, 015501 (2008).

[39] G. Monaco and V. Giordano, Proc. Natl. Acad. Sci. USA 106, 3659 (2009).

[40] G. Monaco and S. Mossa, Proc. Natl. Acad. Sci. USA 106, 16907 (2009).

[41] G. Baldi, V. M. Giordano, G. Monaco, and B. Ruta, Phys. Rev. Lett. 104, 195501 (2010).

[42] G. Baldi, V. M. Giordano, and G. Monaco, Phys. Rev. B 83, 174203 (2011).

[43] B. Ruta, G. Baldi, F. Scarponi, D. Fioretto, V. M. Giordano, and G. Monaco, J. Chem. Phys. 137, 214502 (2012).
[44] U. Buchenau, Condens. Matter Phys. 22, 43601 (2019).

[45] L. D. Landau and E. M. Lifshitz, Theory of Elasticity (Pergamon, Oxford, 1959).

[46] B. Ruzicka, T. Scopigno, S. Caponi, A. Fontana, O. Pilla, P. Giura, G. Monaco, E. Pontecorvo, G. Ruocco, and F. Sette, Phys. Rev. B 69, 100201(R) (2004).

[47] T. Scopigno, E. Pontecorvo, R. Di Leonardo, M. Krisch, G. Monaco, G. Ruocco, B. Ruzicka, and F. Sette, J. Phys.: Condens. Matter 15, S1269 (2003).

[48] G. Baldi, V. M. Giordano, G. Monaco, F. Sette, E. Fabiani, A. Fontana, and G. Ruocco, Phys. Rev. B 77, 214309 (2008).

[49] M. Zanatta, A. Fontana, A. Orecchini, C. Petrillo, and F. Sacchetti, J. Phys. Chem. Lett. 4, 1143 (2013).

[50] A. Cimatoribus, S. Saccani, F. Bencivenga, A. Gessini, M. G. Izzo, and C. Masciovecchio, New J. Phys. 12, 053008 (2010).

[51] F. Bencivenga and D. Antonangeli, Phys. Rev. B 90, 134310 (2014).

[52] D. Bolmatov, M. Zhernenkov, L. Sharpnack, D. M. AgraKooijman, S. Kumar, A. Suvorov, R. Pindak, Y. Q. Cai, and A. Cunsolo, Nano Lett. 17, 3870 (2017).

[53] D. Bolmatov, D. Soloviov, D. Zavýalov, L. Sharpnack, D. M. Agra-Kooijman, and S. Kumar, J. Phys. Chem. Lett. 9, 2546 (2018).

[54] V. Giordano and G. Monaco, Proc. Natl. Acad. Sci. USA 107, 21985 (2010).

[55] M. Sampoli, G. Ruocco, and F. Sette, Phys. Rev. Lett. 79, 1678 (1997).

[56] T. Bryk, G. Ruocco, T. Scopigno, and A. P. Seitsonen, J. Chem. Phys. 143, 104502 (2015).

[57] T. Bryk, T. Demchuk, and N. Jakse, Phys. Rev. B 99, 014201 (2019).

[58] M. C. C. Ribeiro, J. Chem. Phys. 139, 114505 (2013).

[59] R. C. Bourret, Nuovo Cimento 26, 1 (1962).

[60] A. A. Veldhoest and M. C. C. Ribeiro, J. Chem. Phys. 148, 193803 (2018).

[61] K. Yoshimoto, T. S. Jain, K. VanWorkum, P. F. Nealey, and J. J. de Pablo, Phys. Rev. Lett. 93, 175501 (2004).

[62] M. Tsamados, A. Tanguy, C. Goldenberg, and J. L. Barrat, Phys. Rev. E 80, 026112 (2009).

[63] Y. C. Hu, P. F. Guan, M. Z. Li, C. T. Liu, Y. Yang, H. Y. Bai, andW. H. Wang, Phys. Rev. B 93, 214202 (2016).

[64] P. Soven, Phys. Rev. 156, 809 (1967).

[65] D. W. Taylor, Phys. Rev. 156, 1017 (1967).

[66] R. J. Elliott and D. E. Pepper, Phys. Rev. B 8, 2374 (1972).

[67] S. Burdin and P. Fulde, Phys. Rev. B 76, 104425 (2007).

[68] M. A. Korotin, Z. V. Pchelkina, N. A. Skorikov, E. Z. Kurmaev, and V. I. Anisimov, J. Phys.: Condens. Matter 26, 115501 (2014).

[69] R. Zimmermann and C. Schindler, Phys. Rev. B 80, 144202 (2009).

[70] R. J. Elliott, J. A. Krumhansl, and P. L. Leath, Rev. Mod. Phys. 46, 465 (1974).

[71] H. Bruus and K. Flesberg, Many-body Quantum Theory in Condensed Matter Physics (Oxford University Press, Oxford, 2004), and references therein.

[72] R. H. Kraichnan, J. Math. Phys. 2, 124 (1961).

[73] J. Migdal, ZhETF 34, 1438 (1958) [JETP 7, 996 (1958)].

[74] D. Pines, The Many-Body Problem (Benjamin, New York, 1961). 
[75] K. Fujii, R. Kanzaki, T. Takamuku, Y. Kameda, S. Kohara, M. Kanakubo, M. Shibayama, S. Ishiguro, and Y. Umebayashi, J. Chem. Phys. 135, 244502 (2011).

[76] E. Bodo, L. Gontrani, A. Triolo, and R. Caminiti, J. Phys. Chem. Lett. 1, 1095 (2010).

[77] H. V. R. Annapureddy, H. K. Kashyap, P. M. De Biase, and C. J. Margulis, J. Phys. Chem. B 114, 16838 (2010).

[78] F. Ferdeghini, Q. Berrod, J.-M. Zanotti, P. Judeinstein, V. García Sakai, O. Czakkel, P. Fouquetc, and D. Constantind, Nanoscale 9, 1901 (2017).

[79] B. Aoun, A. Goldbach, M. A. González, S. Kohara, D. L. Price, and M. L. Saboungi, J. Chem. Phys. 134, 104509 (2011).

[80] C. Hardacre, J. D. Holbrey, C. L. Mullan, T. G. A. Youngs, and D. T. Bowron, J. Chem. Phys. 133, 074510 (2010).

[81] O. Yamamuro, T. Yamada, M. Kofu, M. Nakakoshi, and M. Nagao, J. Chem. Phys. 135, 054508 (2011).

[82] M. G. Izzo, G. Ruocco, and S. Cazzato, Front. Phys. 6, 108 (2018).

[83] J. Hafner, J. Phys. C: Solid State Phys. 14, L287 (1981).

[84] A. P. Sokolov, U. Buchenau, D. Richter, C. Masciovecchio, F. Sette, A. Mermet, D. Fioretto, G. Ruocco, L. Willner, and B. Frick, Phys. Rev. E 60, R2464 (1999).

[85] U. Borjesson, A. J. Buchenau, D. Dianoux, A. P. Engberg, and A. Sokolov, Phys. B (Amsterdam) 234, 383 (1997).

[86] U. Buchenau, Phys. Rev. E 90, 062319 (2014).

[87] T. Scopigno, M. D'astuto, M. Krisch, G. Ruocco, and F. Sette, Phys. Rev. B 64, 012301 (2001).

[88] See Supplemental Material at http://link.aps.org/supplemental/ 10.1103/PhysRevB.102.214309 for the evaluation of the umklapp scattering contribution to the measured dynamic structure factor of glassy [C8MIMCl]; for computation of the dynamic structure factors in the SCBA framework, including also a possible coupling with IVM, and comparison with experimental data of glassy [C8MIM]Cl; for computation of the dynamic structure factors in the GBA framework including also the spatial fluctuations of the Lamé parameter and comparison with experimental data of glassy [C8MIM]Cl; for a detailed description of how the bending of the dispersion of the characteristic energy of the acousticlike excitations is taken under account and of the definition of the pseudo-Brillouin zone; for an analysis of the dependence from the input parameters of the GBA and a comparison between GBA and Born approximation outputs.

[89] T. C. Penna, L. F. O. Faria, J. R. Matos, and M. C. C. Ribeiro, J. Chem. Phys. 138, 104503 (2013).

[90] M. Kofu, Y. Inamura, Y. Moriya, A. Podlesnyak, G. Ehlers, and O. Yamamuro, J. Mol. Liq. 210, 164 (2015).

[91] E. Fabiani, A. Fontana, and U. Buchenau, J. Chem. Phys. 128, 244507 (2008).
[92] A. Wischnewski, U. Buchenau, A. J. Dianoux, W. A. Kamitakahara, and J. L. Zarestky, Phys. Rev. B 57, 2663 (1998).

[93] J. Jin, J. Li, Y. Liu, X. Q. Li, and Y. Yan, J. Chem. Phys. 140, 244111 (2014).

[94] Z. H. Li, N. H. Tong, X. Zheng, D. Hou, J. H. Wei, J. Hu, and Y. J. Yan, Phys. Rev. Lett. 109, 266403 (2012).

[95] M. Zuniga, J. A. Kong, and L. Tsang, J. Appl. Phys. 51, 2315 (1980).

[96] V. A. Ignatchenko and D. S. Polukhin, J. Phys. A: Math. Theor. 49, 095004 (2016).

[97] A. J. Turner, J. Acoust. Soc. Am. 106, 541 (1999).

[98] A. Bosak, M. Krisch, I. Fischer, S. Huotari, and G. Monaco, Phys. Rev. B 75, 064106 (2007).

[99] T. Ichitsubo, S. Hosokawa, K. Matsuda, E. Matsubara, N. Nishiyama, S. Tsutsui, and A. Q. R. Baron, Phys. Rev. B 76, 140201(R) (2007).

[100] F. Leonforte, R. Boissiere, A. Tanguy, J. P. Wittmer, and J. L. Barrat, Phys. Rev. B 72, 224206 (2005).

[101] M. Dzida, E. Zorebski, M. Zorebski, M. Zarska, M. GeppertRybczynńska, M. Chorazewski, J. Jacquemin, and I. Cibulka, Chem. Rev. 117, 3883 (2017).

[102] J. Lan, W. Yu, and J. Xiao, Nat. Commun. 8, 178 (2017).

[103] W. Yu, J. Lan, and J. Xiao, Phys. Rev. Appl. 13, 024055 (2020).

[104] W. Steurer and D. Sutter, J. Phys. D: Appl. Phys. 40, R229 (2007)

[105] R. E. Cohen, Nature (London) 441, 941 (2006).

[106] M. Eremenko, V. Krayzman, A. Bosak, H. Y. Playford, K. W. Chapman, J. C. Woicik, B. Ravel, and I. Levin, Nat. Commun. 10, 2728 (2019).

[107] A. Bosak, D. Chernyshov, S. Vakhrushev, and M. Krisch, Acta Crystallogr., Sect. A 68, 117 (2012).

[108] W. Schirmacher, G. Ruocco, and V. Mazzone, Phys. Rev. Lett. 115, 015901 (2015).

[109] E. Pontecorvo, M. Krisch, A. Cunsolo, G. Monaco, A. Mermet, R. Verbeni, F. Sette, and G. Ruocco, Phys. Rev. E 71, 011501 (2005).

[110] S. Hosokawa, M. Inui, Y. Kajihara, K. Matsuda, T. Ichitsubo, W.-C. Pilgrim, H. Sinn, L. E. Gonzalez, D. J. Gonzalez, S. Tsutsui, and A. Q. R. Baron, Phys. Rev. Lett. 102, 105502 (2009)

[111] T. Bryk, T. Demchuk, N. Jakse, and J.-F. Wax, Front. Phys. 6, 6 (2018).

[112] B. G. del Rio and L. E. Gonzalez, Phys. Rev. B 95, 224201 (2017).

[113] E. A. Galapon, J. Math. Phys. 57, 033502 (2016).

[114] C. Fox, J. Can. Math. 9, 110 (1957).

[115] J. Baumert, C. Gutt, V. P. Shpakov, J. S. Tse, M. Krisch, M. Muller, H. Requardt, D. D. Klug, S. Janssen, and W. Press, Phys. Rev. B 68, 174301 (2003).

[116] E. Duval, A. Mermet, and L. Saviot, Phys. Rev. B 75, 024201 (2007). 